\title{
AutoPH4: An Automated Method for Generating Pharmacophore Models from Protein Binding Pockets Supporting Information
}

\author{
Siduo Jiang, ${ }^{1}$ Miklos Feher, ${ }^{1, \dagger}$ Chris Williams, ${ }^{2}$ Brian Cole, ${ }^{1}$ and David E. Shaw ${ }^{1,3, \dagger}$ \\ ${ }^{1}$ D. E. Shaw Research, New York, NY 10036, USA. \\ ${ }^{2}$ Chemical Computing Group, Montreal, QC H3A 2R7, Canada. \\ ${ }^{3}$ Department of Biochemistry and Molecular Biophysics, Columbia University, \\ New York, NY 10032, USA.
}

$\dagger$ To whom correspondence should be addressed.

$\underline{\text { Miklos Feher }}$

E-mail: Miklos.Feher@DEShawResearch.com

Phone: (212) 478-0788

Fax: $\quad$ (212) $845-1788$

David E. Shaw

E-mail: David.Shaw@DEShawResearch.com

Phone: (212) 478-0260

Fax: (212) $845-1286$ 


\section{Field settings used for automated pharmacophore feature generation}

For fields available in the Molecular Operating Environment (MOE) package, ${ }^{1}$ the values given here correspond to the default values in MOE.

Table S1. Pharmacophore feature generation with the holo option (r, radius; Metal VDW, van der Waals radius of the metal). Pharmacophore features are placed at the pharmacophore annotation points of the cognate ligands.

\begin{tabular}{|l|l|l|}
\hline Feature & Field & Value \\
\hline Volume of interest & Distance from ligand & $1 \AA$ \\
\hline $\begin{array}{l}\text { Hydrogen bond } \\
\text { acceptor }\end{array}$ & Projected donor points from receptor & $\begin{array}{l}1 \AA \text { sphere around donor points } \\
\text { projected from receptor }\end{array}$ \\
\hline Hydrogen bond donor & Acceptor interaction cones from receptor & $\begin{array}{l}\text { Spherical shells around metal } \\
\text { cations } \\
\text { r }=\left[0.9^{*} \text { Metal VDW }+0.4 \AA\right.\end{array}$ \\
\hline Aromatic & $\begin{array}{l}\text { Contact statistics } \\
\text { Cation cone from receptor }\end{array}$ & $\begin{array}{l}25 \% \\
0.5 \text { (arbitrary units); spherical } \\
\text { shells } 1.8-3.5 \AA \text { around } \mathrm{N}^{+} \text {atoms } \\
\text { in lysine and arginine }\end{array}$ \\
\hline Hydrophobe & $\begin{array}{l}\text { (Alternative to contact statistics: Poisson- } \\
\text { Boltzmann electrostatics) }\end{array}$ & $\begin{array}{l}-2 \\
\text { Poisson-Boltzmann electrostatics }\end{array}$ \\
\hline
\end{tabular}


Table S2. Pharmacophore feature generation with the apo option (r, radius; Metal VDW, van der Waals radius of the metal). Pharmacophore features are placed as determined from the clustering procedure.

\begin{tabular}{|c|c|c|}
\hline Feature & Field & Value \\
\hline Volume of interest & $\begin{array}{l}\text { Can be defined as distance from dummy } \\
\text { atoms (e.g., from Site Finder) or ligands, } \\
\text { or alternatively as pocket atoms in MOE, } \\
\text { as a set of selected atoms, or as a set of } \\
\text { selected residues }\end{array}$ & $\begin{array}{l}1 \AA \text { from ligands or } \\
\text { dummy atoms } \\
\text { Full residues with any } \\
\text { atom within } 4.5 \AA \text { of } \\
\text { selection are considered } \\
\text { for feature generation }\end{array}$ \\
\hline \multirow[t]{2}{*}{ Hydrogen bond acceptor } & GRID probe & $\begin{array}{l}\text { Probe: O (oxygen atom) } \\
\text { Level 1: }-4 \mathrm{kcal} / \mathrm{mol}\end{array}$ \\
\hline & $\begin{array}{l}\text { (Alternative: } \\
\text { projected donor points from receptor }+ \\
\text { coordination sphere from metal atoms) }\end{array}$ & $\begin{array}{l}1 \AA \text { sphere around donor } \\
\text { points projected from } \\
\text { receptor combined with } \\
\text { spherical shells around } \\
\text { metal cation } r=\left[0.9^{*}\right. \\
\text { Metal VDW, Metal VDW } \\
+0.4 \AA]\end{array}$ \\
\hline \multirow[t]{2}{*}{ Hydrogen bond donor } & GRID probe & $\begin{array}{l}\text { Probe: }{ }^{\mathrm{a}} \mathrm{Na}^{+} \\
\text {Level: }^{\mathrm{a}}-3 \mathrm{kcal} / \mathrm{mol}\end{array}$ \\
\hline & $\begin{array}{l}\text { (Alternative 1: projected acceptor points } \\
\text { from receptor) } \\
\text { (Alternative } 2 \text { : contact statistics) }\end{array}$ & $\begin{array}{l}1.5 \text { (arbitrary units) } \\
95 \%\end{array}$ \\
\hline \multirow[t]{2}{*}{ Aromatic feature } & Fast Fourier Transform (FFT) probe & $-0.14 \mathrm{kcal} / \mathrm{mol}$ \\
\hline & (Alternative: contact statistics) & $25 \%$ \\
\hline Hydrophobe & Poisson-Boltzmann electrostatics & $-3 \mathrm{kcal} / \mathrm{mol}$ \\
\hline
\end{tabular}


a These grid levels were used in all results described in this work. They worked well for most receptors, but might be suboptimal for some. In cases where the pocket was very polar, for example, we found in experiments that toning down the grid level and changing to a $\mathrm{N}^{+}$probe improved the results. For carbonic anhydrases, the optimal grid probe was $\mathrm{N}^{+}$with a donor grid level of $-5 \mathrm{kcal} / \mathrm{mol}$, and the level for the $\mathrm{O}$ acceptor probe was $-6 \mathrm{kcal} / \mathrm{mol}$. Similarly, for ionotropic glutamates, the optimal donor probe would be $\mathrm{N}^{+}$with both grid levels set to $-6 \mathrm{kcal} / \mathrm{mol}$. Although for simplicity we did not use such target-class specific parameters to obtain the results reported this work, it may be possible to take advantage of them in the future. 
Table S3. Feature diameters and clustering radii (in $\AA$ ) for the A) holo and B) apo options.

A
\begin{tabular}{|l|l|l|l|}
\hline Feature & Minimum radius & Maximum radius & Clustering radius \\
\hline Hydrogen bond acceptor & 1.2 & 1.2 & 1.0 \\
\hline Hydrogen bond donor & 1.2 & 1.2 & 1.0 \\
\hline Aromatic & 1.4 & 1.4 & 1.0 \\
\hline Hydrophobe & 1.6 & 3.0 & 1.0 \\
\hline
\end{tabular}

B

\begin{tabular}{|l|l|l|l|}
\hline Feature & Minimum radius & Maximum radius & Clustering radius \\
\hline Hydrogen bond acceptor & 1.4 & 1.4 & 1.5 \\
\hline Hydrogen bond donor & 1.4 & 1.4 & 1.0 \\
\hline Aromatic & 1.6 & 1.6 & 1.5 \\
\hline Hydrophobe & 1.6 & 3.0 & 1.5 \\
\hline
\end{tabular}


Comparison of our AutoPH4-based workflow, Glide, GOLD, and ECFP4 fingerprints as applied to three targets

We show results for three example targets: heat shock protein, androgen receptor, and cyclin-dependent kinase 2 .

\section{Heat shock protein}

The dataset for heat shock protein (PDB code: 1UYG, DUD-E code: hs90a) contained 4,936 molecules, 88 of which were actives. 
Table S4. Correlation in ranking of the AutoPH4-based workflow and docking methods on the heat shock protein, a member of the DUD-E dataset. Results are shown separately for all molecules (i.e., DUD-E actives and decoys for this target) as well as for DUD-E actives alone. A) number of molecules that were scored by each of a given pair of methods (typically slightly less than the total number of molecules as it may not be possible to dock certain molecules using a given method), B) Kendall rank correlations for all scored molecules, C) number of DUD-E actives scored by each of a pair of methods, and D) Kendall rank correlations for the actives.

A

\begin{tabular}{|l|l|l|l|l|}
\hline & Glide & GOLD & AutoPH4 $^{\text {a }}$ & ECFP4 \\
\hline Glide & 3,821 & 3,390 & 3,807 & 3,821 \\
\hline GOLD & & 4,414 & 4,404 & 4,414 \\
\hline AutoPH4 ${ }^{\text {a }}$ & & & 4,922 & 4,922 \\
\hline ECFP4 & & & & 4,936 \\
\hline
\end{tabular}

B

\begin{tabular}{|l|l|l|l|}
\hline & GOLD & AutoPH4 $^{\text {a }}$ & ECFP4 \\
\hline Glide & -0.064 & -0.028 & -0.035 \\
\hline GOLD & & 0.189 & 0.010 \\
\hline AutoPH4 $^{\text {a }}$ & & & 0.151 \\
\hline
\end{tabular}

C

\begin{tabular}{|c|c|c|c|c|}
\hline & Glide & GOLD & AutoPH4 ${ }^{a}$ & ECFP4 \\
\hline Glide & 86 & 73 & 86 & 86 \\
\hline GOLD & & 75 & 75 & 75 \\
\hline AutoPH4 ${ }^{\mathrm{a}}$ & & & 88 & 88 \\
\hline ECFP4 & & & & 88 \\
\hline
\end{tabular}

D

\begin{tabular}{|l|l|l|l|}
\hline & GOLD & AutoPH4 $^{\text {a }}$ & ECFP4 \\
\hline Glide & -0.090 & -0.065 & -0.118 \\
\hline GOLD & & 0.545 & 0.241 \\
\hline AutoPH4 $^{\text {a }}$ & & & 0.314 \\
\hline
\end{tabular}

a Here "AutoPH4" denotes the AutoPH4-based virtual screening workflow. 
Androgen receptor

The dataset for the androgen receptor (PDB code:2AM9, DUD-E code: andr) contained 14,612 molecules, 269 of which were active. 
Table S5. Correlation in ranking of the AutoPH4-based workflow and docking methods on the androgen receptor, a member of the DUD-E dataset. Results are shown separately for all molecules (i.e., DUD-E actives and decoys for this target) as well as for DUD-E actives alone. A) number of molecules that were scored by each of a given pair of methods (typically slightly less than the total number of molecules as it may not be possible to dock certain molecules using a given method), B) Kendall rank correlations for all scored molecules, C) number of DUD-E actives scored by each of a pair of methods, and D) Kendall rank correlations for the actives.

A

\begin{tabular}{|l|l|l|l|l|}
\hline & Glide & GOLD & AutoPH4 $^{\text {a }}$ & ECFP4 \\
\hline Glide & 9,261 & 8,209 & 8,192 & 9,261 \\
\hline GOLD & & 11,752 & 10,139 & 11,752 \\
\hline AutoPH4 ${ }^{\text {a }}$ & & & 12,701 & 12,701 \\
\hline ECFP4 & & & & 14,612 \\
\hline
\end{tabular}

B

\begin{tabular}{|l|l|l|l|}
\hline & GOLD & AutoPH4 $^{\text {a }}$ & ECFP4 \\
\hline Glide & 0.286 & 0.332 & 0.054 \\
\hline GOLD & & 0.188 & -0.127 \\
\hline AutoPH4 $^{\text {a }}$ & & & 0.012 \\
\hline
\end{tabular}

$\mathrm{C}$

\begin{tabular}{|c|c|c|c|c|}
\hline & Glide & GOLD & AutoPH4 ${ }^{a}$ & ECFP4 \\
\hline Glide & 164 & 151 & 152 & 164 \\
\hline GOLD & & 211 & 187 & 211 \\
\hline AutoPH4 ${ }^{\mathrm{a}}$ & & & 239 & 239 \\
\hline ECFP4 & & & & 269 \\
\hline
\end{tabular}

$\mathrm{D}$

\begin{tabular}{|l|l|l|l|}
\hline & GOLD & AutoPH4 $^{\text {a }}$ & ECFP4 \\
\hline Glide & 0.486 & 0.497 & 0.051 \\
\hline GOLD & & 0.346 & 0.015 \\
\hline AutoPH4 $^{\text {a }}$ & & & 0.050 \\
\hline
\end{tabular}

a Here "AutoPH4" denotes the AutoPH4-based virtual screening workflow. 


\section{Cyclin-dependent kinase 2}

The dataset for cyclin-dependent kinase 2 (PDB code:1H00, DUD-E code: cdk2) contained 28,304 molecules, of which 474 were active. 
Table S6. Correlation in ranking of the AutoPH4-based workflow and docking methods on the cyclin dependent kinase 2, a member of the DUD-E dataset. Results are shown separately for all molecules (i.e., DUD-E actives and decoys for this target) as well as for DUD-E actives alone. A) number of molecules that were scored by each of a given pair of methods (typically slightly less than the total number of molecules as it may not be possible to dock certain molecules using a given method), B) Kendall rank correlations for all scored molecules, C) number of DUD-E actives scored by each of a pair of methods, and D) Kendall rank correlations for the actives.

A

\begin{tabular}{|l|l|l|l|l|}
\hline & Glide & GOLD & AutoPH4 $^{\text {a }}$ & ECFP4 \\
\hline Glide & 27,997 & 27,954 & 27,521 & 27,997 \\
\hline GOLD & & 28,260 & 27,750 & 28,260 \\
\hline AutoPH4 ${ }^{\text {a }}$ & & & 27,789 & 27,789 \\
\hline ECFP4 & & & & 28,304 \\
\hline
\end{tabular}

B

\begin{tabular}{|l|l|l|l|}
\hline & GOLD & AutoPH4 $^{\text {a }}$ & ECFP4 \\
\hline Glide & 0.158 & 0.242 & 0.088 \\
\hline GOLD & & 0.212 & 0.157 \\
\hline AutoPH4 $^{\text {a }}$ & & & 0.149 \\
\hline
\end{tabular}

$\mathrm{C}$

\begin{tabular}{|c|c|c|c|c|}
\hline & Glide & GOLD & AutoPH4 ${ }^{a}$ & ECFP4 \\
\hline Glide & 471 & 471 & 471 & 471 \\
\hline GOLD & & 474 & 474 & 474 \\
\hline AutoPH4 ${ }^{\mathrm{a}}$ & & & 474 & 474 \\
\hline ECFP4 & & & & 474 \\
\hline
\end{tabular}

$\mathrm{D}$

\begin{tabular}{|l|l|l|l|}
\hline & GOLD & AutoPH4 $^{\text {a }}$ & ECFP4 \\
\hline Glide & 0.126 & 0.207 & -0.050 \\
\hline GOLD & & 0.131 & 0.162 \\
\hline AutoPH4 $^{\text {a }}$ & & & 0.110 \\
\hline
\end{tabular}

a Here "AutoPH4" denotes the AutoPH4-based virtual screening workflow. 


\section{Performance of the AutoPH4-based virtual screening workflow}

Performance of the AutoPH4-based workflow by holo/apo option and by force field

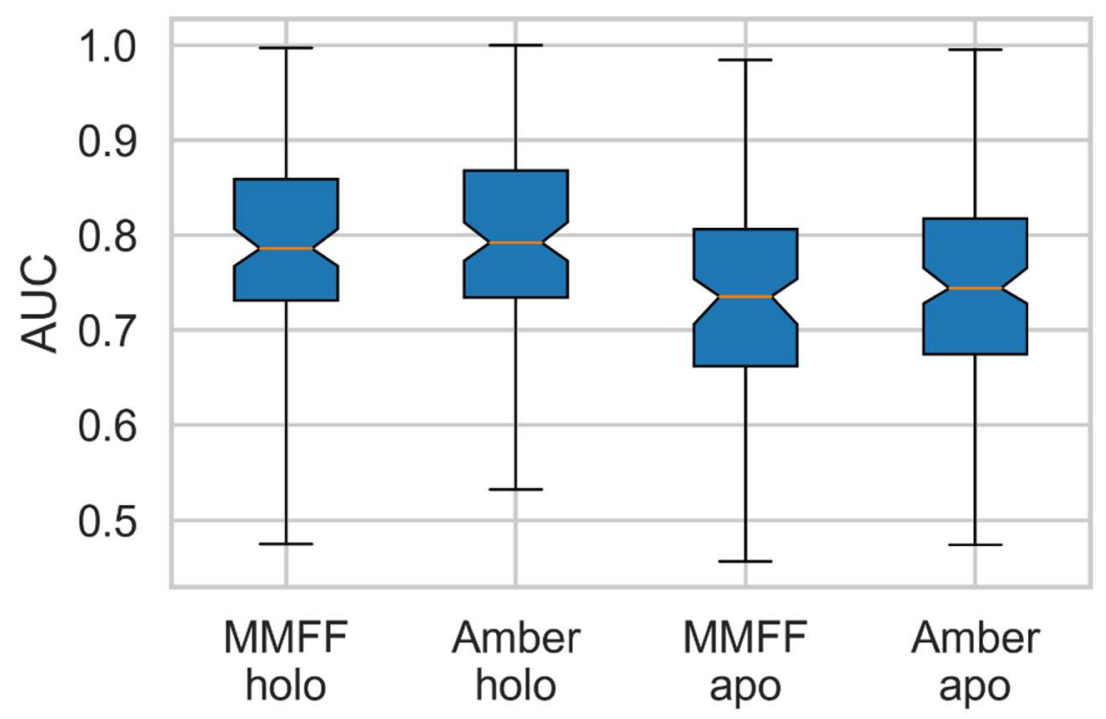

Figure S1. Area under the curve (AUC) for the virtual screening workflow using AutoPH4 with the holo and apo options, and with the MMFF94x and Amber14-EHT force fields implemented in MOE on the DUD-E data set $(n=94)$. Whiskers represent minimum and maximum values; notches represent the standard error (corresponding to a $95 \%$ confidence interval) of the median (orange lines), obtained from bootstrapping. 
Performance of the AutoPH4-based workflow compared to other virtual screening methods

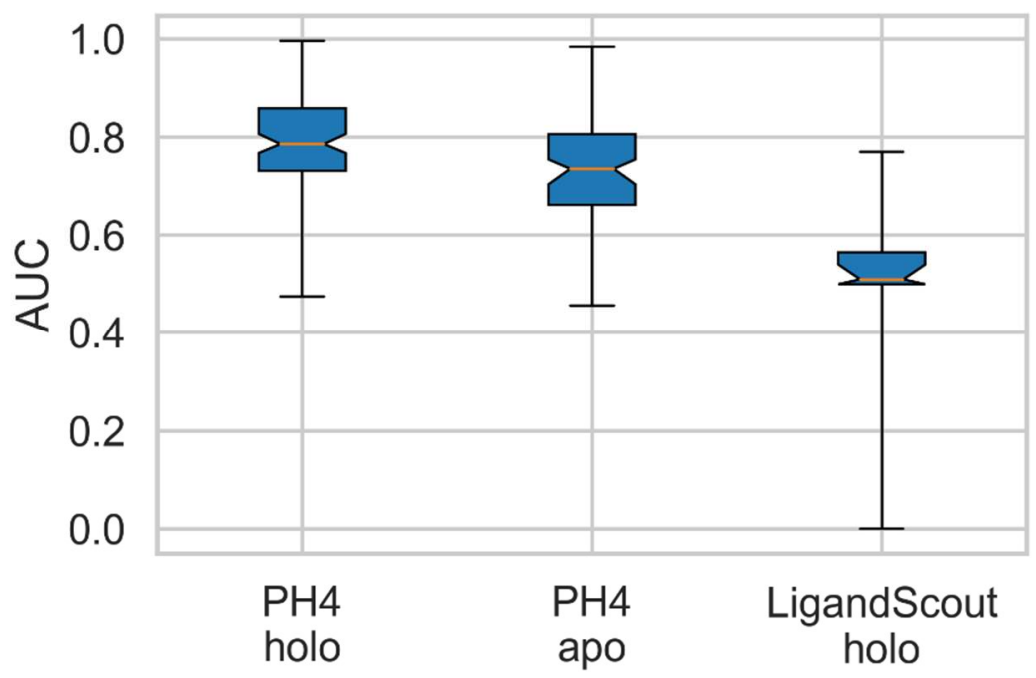

Figure S2. Area under the curve (AUC) for the AutoPH4-based virtual screening workflow ("PH4" on X-axis) and LigandScout on data common to both our work and Table 2 of the paper by Wieder et al. ${ }^{2}(n=38)$. In this analysis, we replaced the zero AUC values from Table 2 in the Wieder et al. paper with the correct value deduced from their Figure 5. (We note that in the Wieder et al. paper, for two of the common targets (hivpr and gcr), LigandScout encountered difficulties when used with the original the DUD-E complexes, which were then replaced by different PDB complexes.) Whiskers represent maximum and minimum values; notches represent the standard error (corresponding to a 95\% confidence interval), obtained from bootstrapping. 


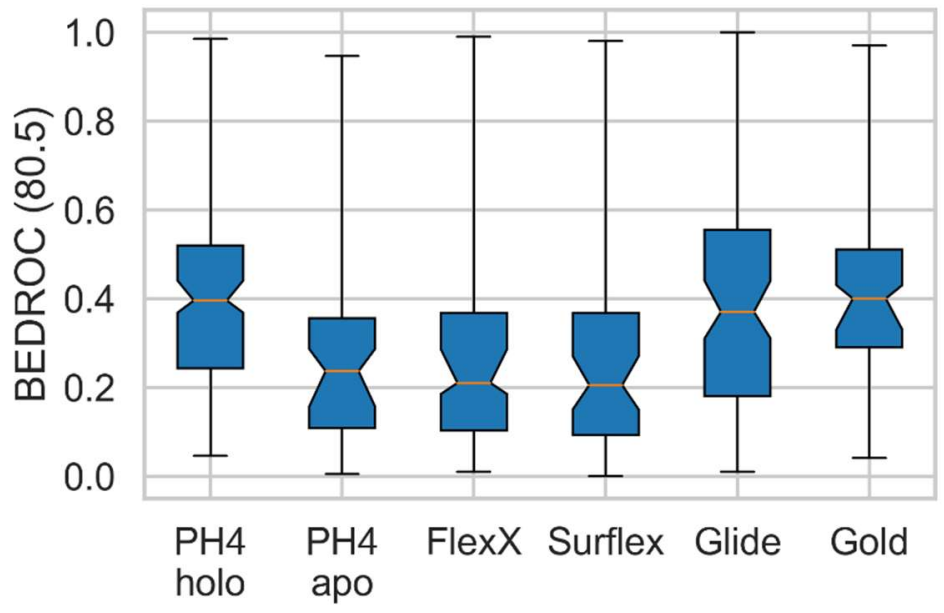

Figure S3. Boltzmann-enhanced discrimination of the receiver operating characteristic (BEDROC) scores (with adjustable parameter $\alpha=80.5$ ) for the AutoPH4-based virtual screening workflow ("PH4" on x-axis) and other methods (FlexX, Surflex, Glide, and Gold) on data common to our work and Table S1 of the paper by Chaput et al. ${ }^{3}(\mathrm{n}=94)$. Whiskers represent maximum and minimum values; notches represent the standard error (corresponding to a $95 \%$ confidence interval), obtained from bootstrapping. 
A

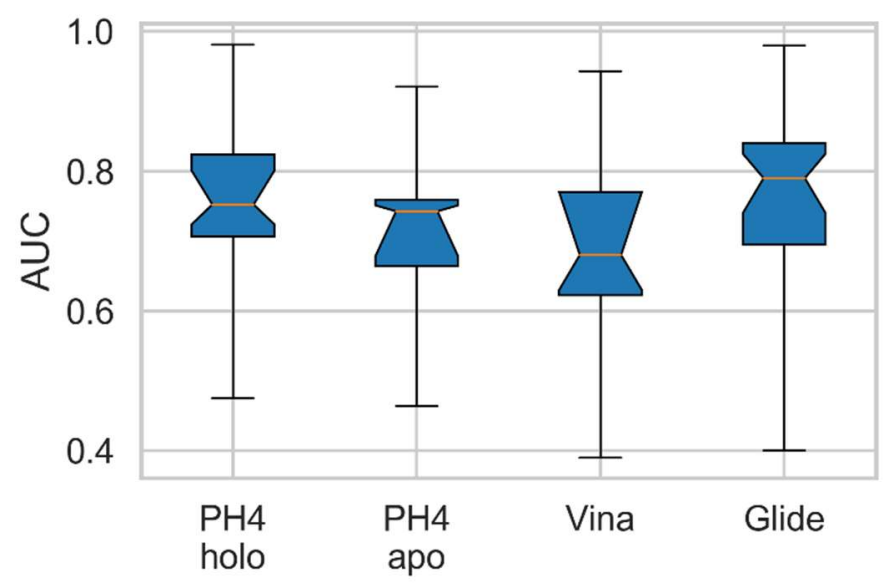

$\mathrm{B}$

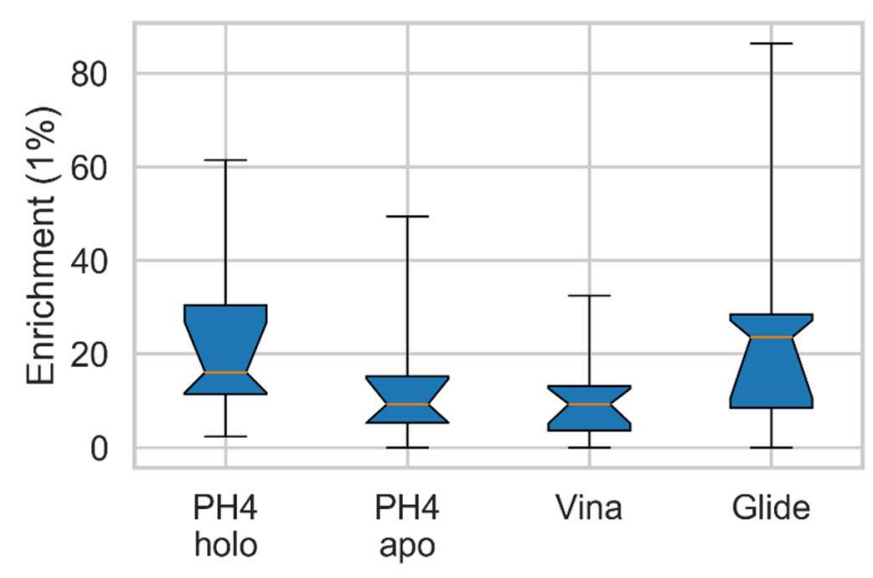

$\mathrm{C}$

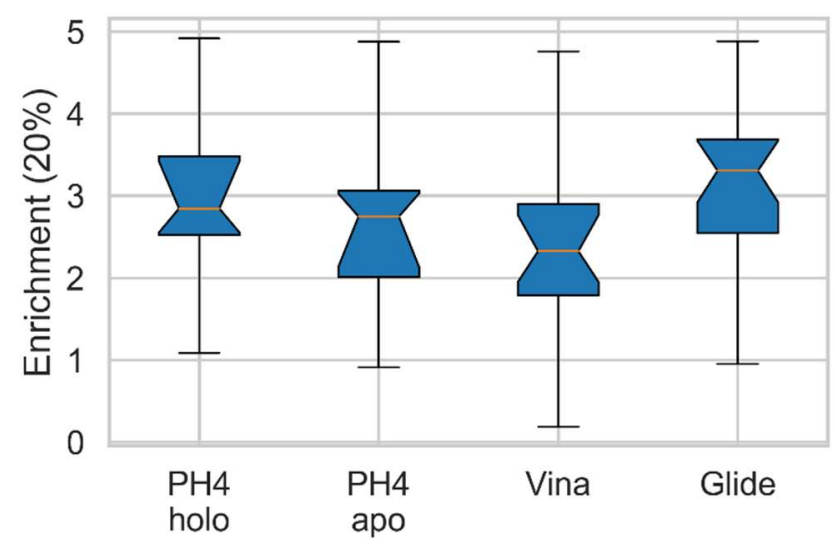

Figure S4. Performance, in terms of A) area under the curve (AUC), B) 1\% enrichment, and C) $20 \%$ enrichment, of the AutoPH4-based virtual screening workflow ("PH4" on the $\mathrm{x}$-axis) and other methods (Vina and Glide) on data common to our work and Table S1 of the paper by Ruiz-Carmona et al. ${ }^{4}(\mathrm{n}=22)$. Whiskers represent maximum and minimum values; notches represent the standard error (corresponding to a $95 \%$ confidence interval), obtained from bootstrapping. 


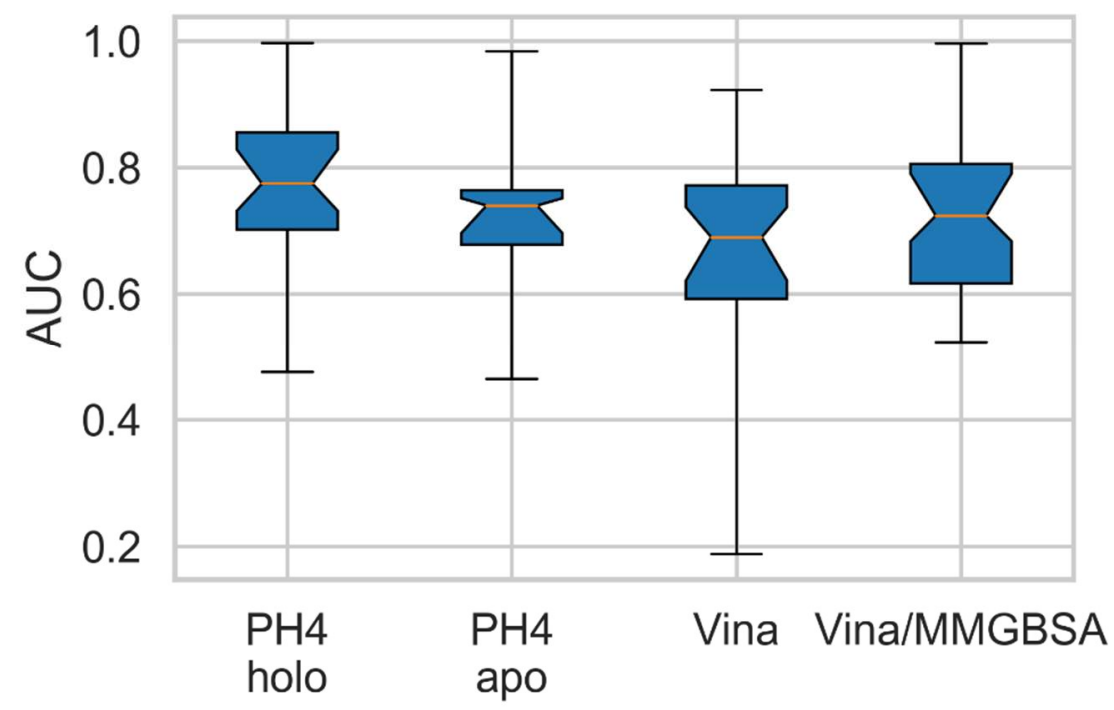

Figure S5. Area under the curve (AUC) for the AutoPH4-based virtual screening workflow ("PH4" on x-axis) and AutoDock Vina, both with default options and with the molecular mechanics/generalized Born surface area (MM/GBSA) measure of binding affinity, on data common to our work and Table S1 of the paper by Zhang et al. ${ }^{5}(\mathrm{n}=34)$. Whiskers represent maximum and minimum values; notches represent the standard error (corresponding to a $95 \%$ confidence interval), obtained from bootstrapping. 
A

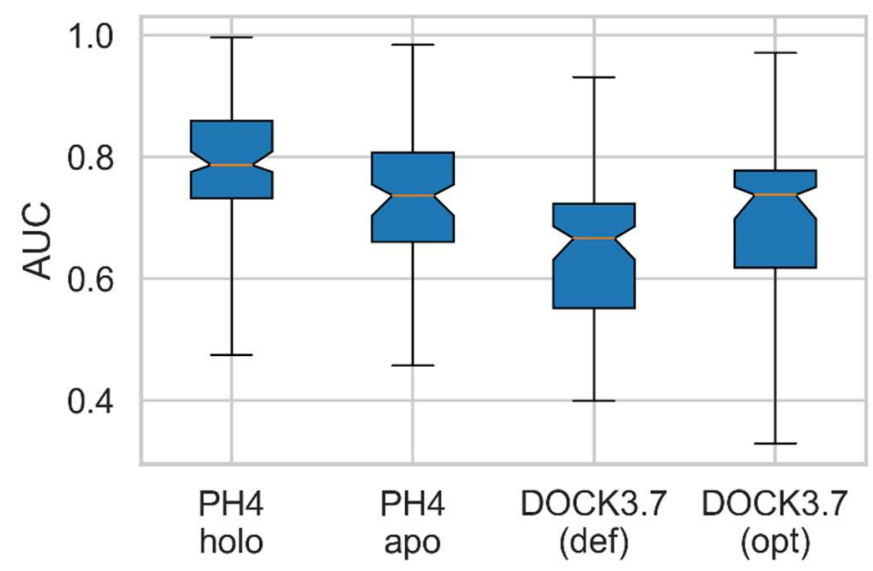

$\mathrm{B}$

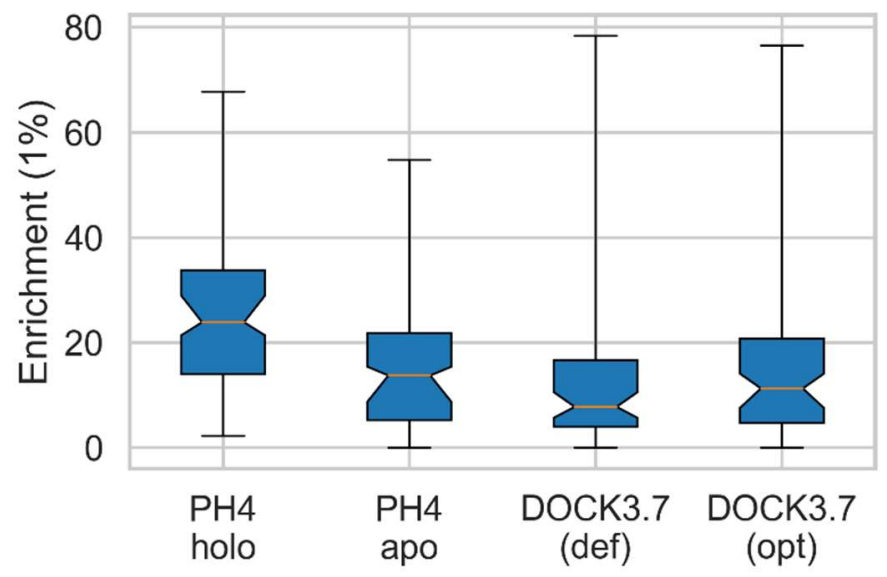

Figure S6. Performance in terms of A) area under the curve (AUC) and B) 1\% enrichment, for the AutoPH4-based virtual screening workflow ("PH4" on X-axis) and DOCK3.7, both with the 0.33 / 50E column corresponding to default settings ("def") and with the column giving the best results ("opt"), on data ( $\mathrm{n}=94)$ presented in the SI of the paper by Coleman et al. $^{6}(\mathrm{n}=94)$. Whiskers represent maximum and minimum values; notches represent the standard error (corresponding to a $95 \%$ confidence interval), obtained from bootstrapping. 
A

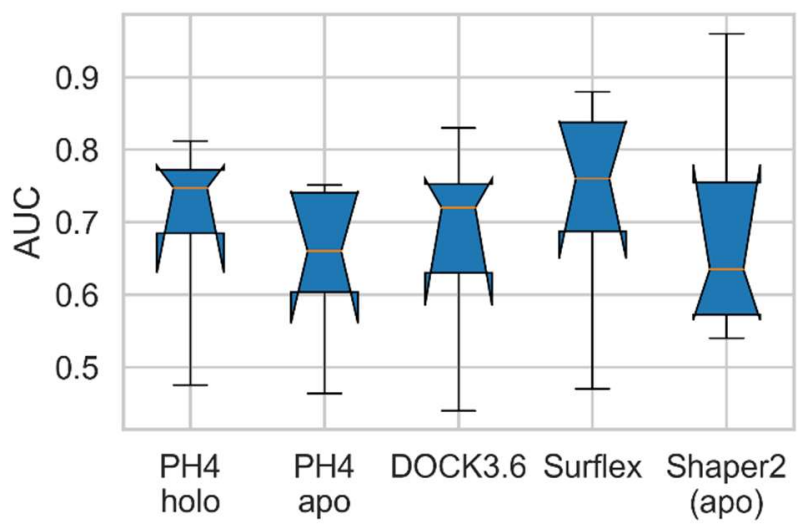

C

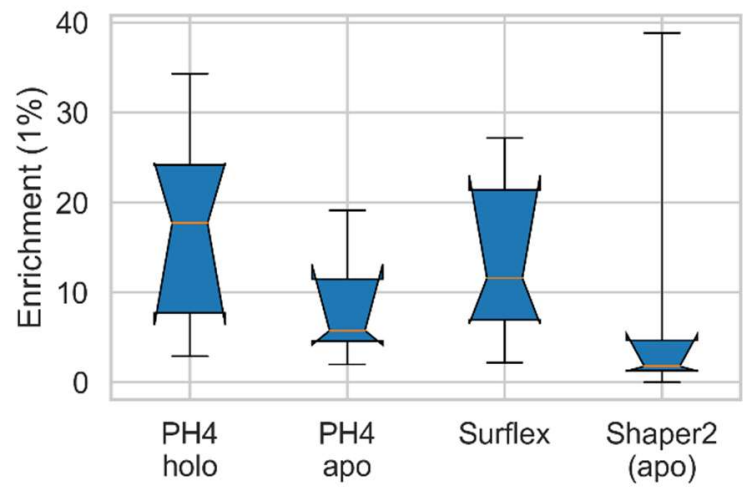

B

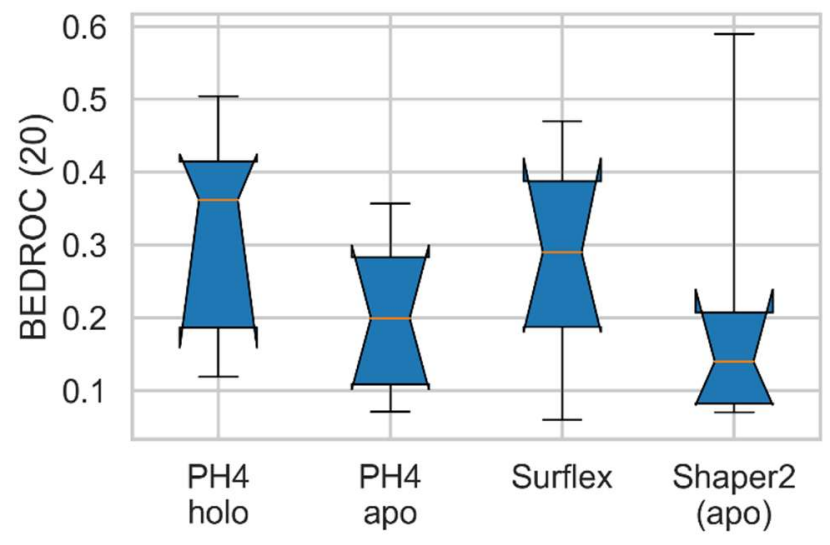

Figure S7. Performance in terms of A) area under the curve (AUC) and B) BEDROC $(\alpha=20)$ and C) $1 \%$ enrichment, for the AutoPH4-based virtual screening workflow ("PH4 holo" and "PH4 apo" on $x$-axis), DOCK 3.6 (for which only AUC data are available), Surflex and Shaper2 with the "TotE PLP" settings on data $(\mathrm{n}=10)$ presented in the paper by Tran-Nguyen et al. ${ }^{7}$ (We note that in the Tran-Nguyen et al. paper, some of the original the DUD-E complexes were replaced by different PDB complexes.) Whiskers represent maximum and minimum values; notches represent the standard error (corresponding to a $95 \%$ confidence interval), obtained from bootstrapping. 
Pharmacophore placement with AutoPH4 in the virtual screening workflow

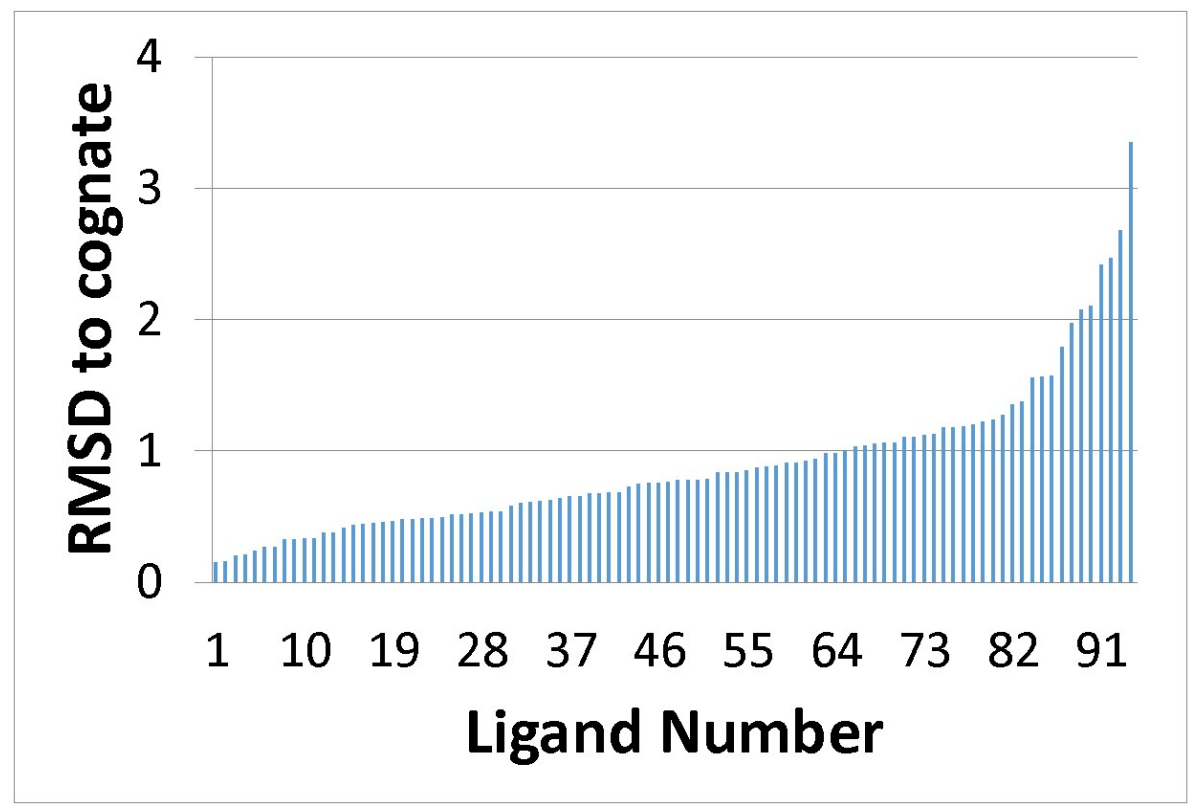

Figure S8. Distribution of root mean squared distances (RMSD, in $\AA$ ) of each cognate ligand in the DUD-E set between the x-ray structure and the placed pharmacophore (with the holo option and before MMFF94x minimization). 


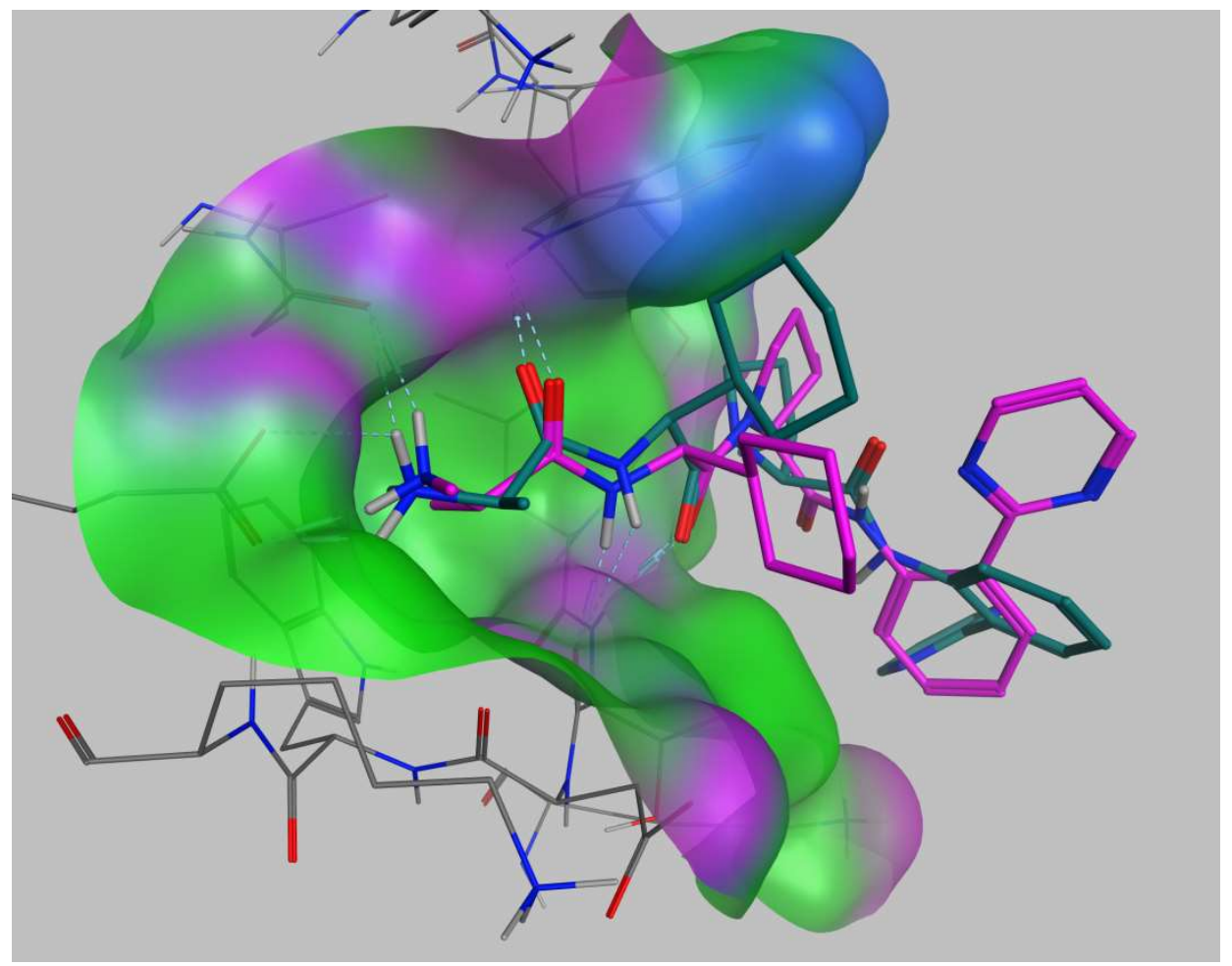

Figure S9. Placement of the cognate ligand (teal stick representation) using an AutoPH4generated pharmacophore model, compared with the x-ray structure (magenta stick representation) for the X-linked inhibitor of apoptosis protein (XIAP-BIR3, DUD-E code: xiap, PDB code: 3HL5). The molecular (Conolly) surface of the protein is colored by property: hydrophobic (green), hydrogen-bonding (purple), and mild polar (blue). The solvent-exposed portion of the placed ligand is largely responsible for an overall root mean squared distance of $3.3 \AA$, corresponding to the largest deviation in Figure S7. The portion of the ligand in contact with the target is placed almost identically to that of the x-ray structure and establishes the same hydrogen-bonding interactions. 
A

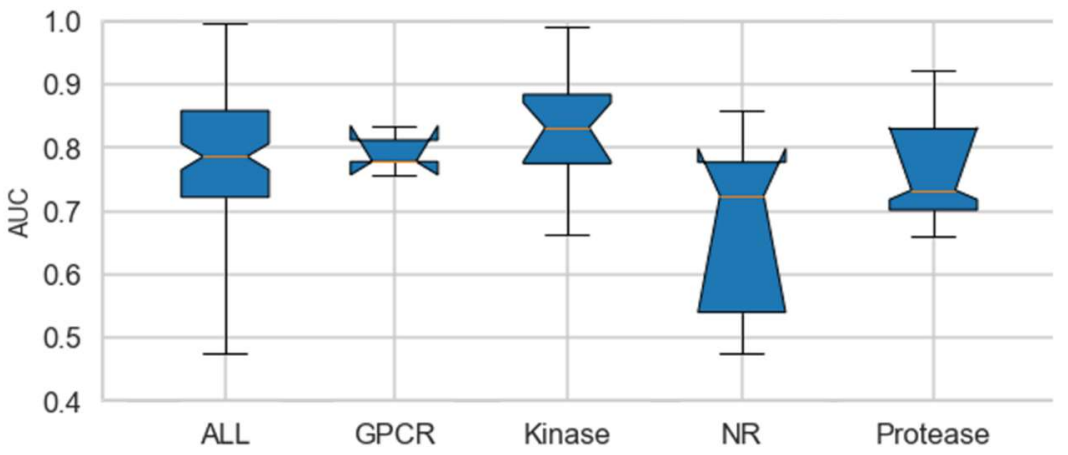

B

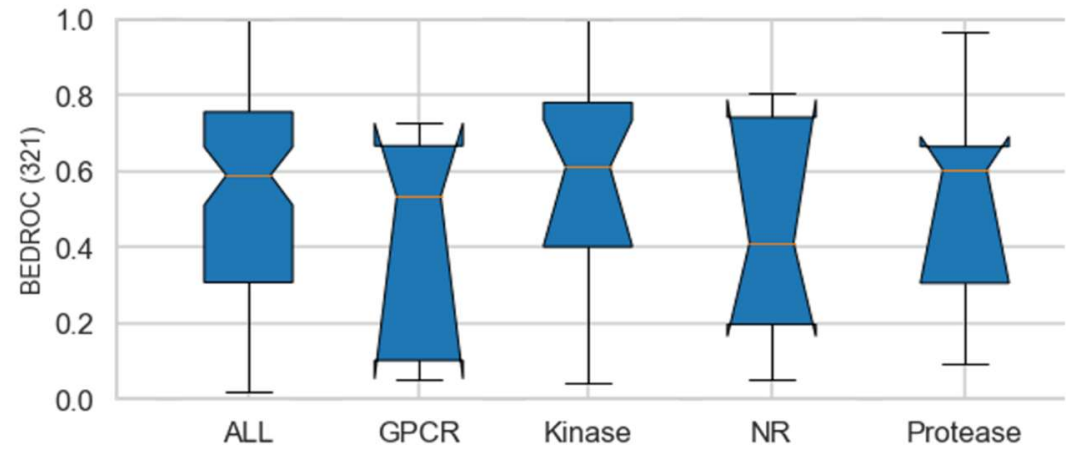

C

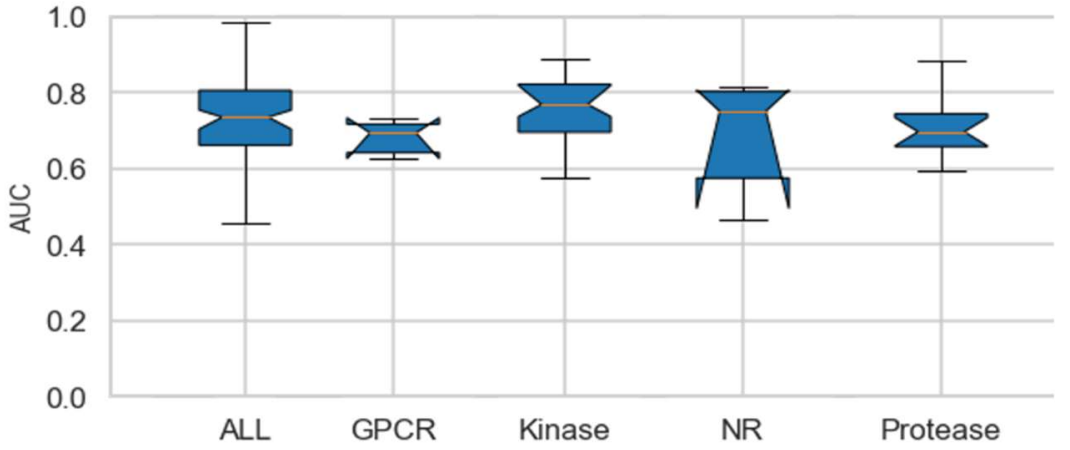

D

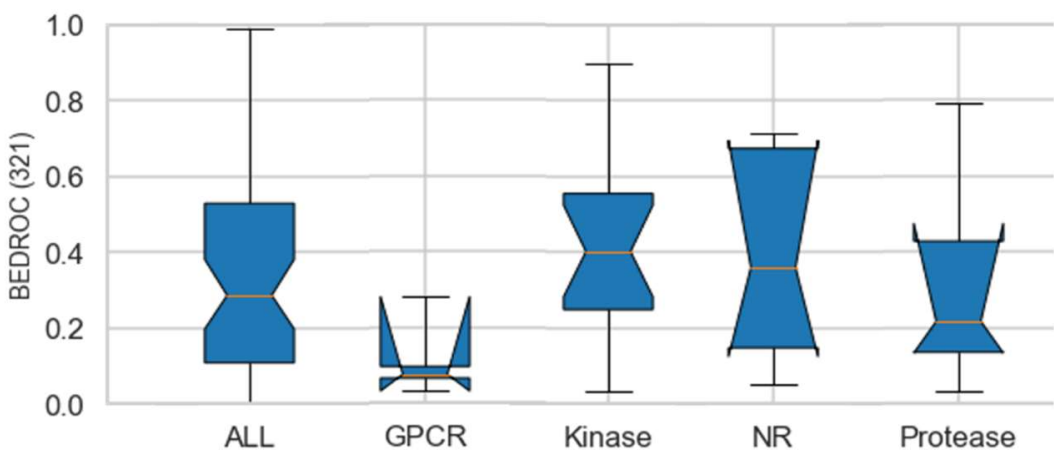


Figure S10. Area under the curve (AUC) for the virtual screening workflow using AutoPH4 with the holo and apo options for different target families on the DUD-E dataset ( $\mathrm{n}=94)$. A) holo case, AUC; B) holo case, BEDROC ( $\alpha=321)$; C) apo case, AUC; D) apo case, BEDROC $(\alpha=321)$. The classification of targets into families corresponds to that on the DUD-E website. These tests were performed using the MMFF94x force field implemented in MOE. Whiskers represent minimum and maximum values; notches represent the standard error (corresponding to a 95\% confidence interval) of the median (orange lines), obtained from bootstrapping. 
Performance of the AutoPH4-based virtual screening workflow by complex

Table S7. Performance of the AutoPH4-based virtual screening method by complex: area under the curve (AUC), enrichment factor (EF) for different percentages of the ranked data set, and Boltzmann-enhanced discrimination of the receiver operating characteristic (BEDROC) values with different values of the adjustable parameter $\alpha$, for each complex in the DUD-E test set using pharmacophore models generated by AutoPH4, with A) the holo option, B) the holo option using only pharmacophore scoring (i.e., no minimization and no MM/GBVI component), and C) the apo option.

A

\begin{tabular}{|c|c|c|c|c|c|c|c|c|c|}
\hline Complex & AUC & $\begin{array}{c}\text { EF } \\
0.5 \%\end{array}$ & $\begin{array}{c}\text { EF } \\
1 \%\end{array}$ & $\begin{array}{c}\text { EF } \\
2 \%\end{array}$ & $\begin{array}{c}\text { EF } \\
8 \%\end{array}$ & $\begin{array}{c}\text { EF } \\
20 \%\end{array}$ & $\begin{array}{c}\text { BEDROC } \\
\alpha=20\end{array}$ & $\begin{array}{c}\text { BEDROC } \\
\alpha=80.5\end{array}$ & $\begin{array}{c}\text { BEDROC } \\
\alpha=321.0\end{array}$ \\
\hline aa2ar & 0.778 & 48.28 & 34.34 & 20.95 & 6.61 & 3.19 & 0.504 & 0.507 & 0.727 \\
\hline ab11 & 0.787 & 33.84 & 22.58 & 13.71 & 6.32 & 2.91 & 0.423 & 0.378 & 0.611 \\
\hline ace & 0.673 & 16.23 & 11.71 & 8.52 & 4.03 & 2.54 & 0.272 & 0.21 & 0.303 \\
\hline aces & 0.591 & 34.53 & 18.52 & 9.61 & 3.09 & 1.87 & 0.255 & 0.303 & 0.61 \\
\hline ada & 0.793 & 40.44 & 24.92 & 14.5 & 5.65 & 3.39 & 0.427 & 0.414 & 0.685 \\
\hline ada17 & 0.832 & 37.89 & 33.67 & 23.01 & 7.73 & 3.61 & 0.552 & 0.495 & 0.578 \\
\hline adrb1 & 0.835 & 45.59 & 33.17 & 20.43 & 7.95 & 3.58 & 0.56 & 0.509 & 0.667 \\
\hline adrb2 & 0.812 & 34.68 & 24.27 & 15.17 & 6.22 & 3.2 & 0.425 & 0.38 & 0.534 \\
\hline akt1 & 0.755 & 15.61 & 14 & 10.41 & 5.16 & 3.26 & 0.349 & 0.26 & 0.331 \\
\hline akt2 & 0.778 & 23.96 & 18.83 & 12.41 & 5.45 & 3.12 & 0.381 & 0.313 & 0.435 \\
\hline aldr & 0.781 & 45.01 & 30.34 & 18.86 & 6.21 & 3.14 & 0.481 & 0.499 & 0.767 \\
\hline ampc & 0.801 & 21.43 & 16.55 & 15.52 & 6.26 & 3.23 & 0.422 & 0.323 & 0.346 \\
\hline andr & 0.54 & 10.45 & 6.35 & 4.29 & 2.05 & 1.49 & 0.144 & 0.117 & 0.197 \\
\hline bace1 & 0.721 & 14.1 & 9.87 & 7.95 & 3.84 & 2.53 & 0.246 & 0.176 & 0.227 \\
\hline braf & 0.884 & 31.88 & 24.33 & 19.07 & 7.15 & 3.95 & 0.503 & 0.416 & 0.522 \\
\hline cah2 & 0.686 & 8.13 & 7.12 & 5.9 & 3.86 & 2.6 & 0.228 & 0.125 & 0.118 \\
\hline casp3 & 0.666 & 32.42 & 19.57 & 12.55 & 4.71 & 2.26 & 0.349 & 0.356 & 0.604 \\
\hline cdk2 & 0.832 & 36.16 & 26.8 & 17.83 & 6.96 & 3.5 & 0.491 & 0.445 & 0.614 \\
\hline comt & 0.95 & 29.95 & 31.62 & 20.67 & 9.76 & 4.88 & 0.548 & 0.378 & 0.354 \\
\hline cp2c9 & 0.58 & 1.66 & 2.49 & 1.67 & 1.77 & 1.5 & 0.096 & 0.037 & 0.022 \\
\hline cp3a4 & 0.686 & 9.39 & 6.45 & 5.3 & 3.16 & 2.21 & 0.184 & 0.11 & 0.116 \\
\hline csf1r & 0.809 & 51.43 & 30.14 & 17.48 & 5.65 & 3.13 & 0.431 & 0.433 & 0.667 \\
\hline cxcr4 & 0.757 & 5.07 & 5.07 & 3.75 & 3.75 & 3.13 & 0.18 & 0.068 & 0.053 \\
\hline
\end{tabular}




\begin{tabular}{|c|c|c|c|c|c|c|c|c|c|}
\hline def & 0.785 & 15.68 & 21.56 & 20.58 & 6.98 & 3.09 & 0.5 & 0.407 & 0.261 \\
\hline dhil & 0.528 & 9.12 & 5.45 & 3.64 & 2.01 & 1.44 & 0.133 & 0.097 & 0.134 \\
\hline dpp4 & 0.733 & 48.17 & 30.29 & 17.76 & 5.76 & 2.91 & 0.438 & 0.432 & 0.638 \\
\hline drd3 & 0.778 & 8.35 & 9.58 & 8.95 & 5 & 3.08 & 0.294 & 0.162 & 0.102 \\
\hline dyr & 0.889 & 75.31 & 61.46 & 34.84 & 9.85 & 4.22 & 0.772 & 0.784 & 0.933 \\
\hline egfr & 0.859 & 47.63 & 33.78 & 22.28 & 7.74 & 3.95 & 0.566 & 0.527 & 0.721 \\
\hline esr1 & 0.691 & 47.59 & 32.16 & 18.39 & 5.71 & 2.65 & 0.457 & 0.513 & 0.804 \\
\hline esr2 & 0.777 & 46.73 & 30.57 & 18.79 & 7.08 & 3.3 & 0.52 & 0.519 & 0.789 \\
\hline fa10 & 0.685 & 29.82 & 18.63 & 11.95 & 4.29 & 2.41 & 0.321 & 0.331 & 0.581 \\
\hline $\mathrm{fa} 7$ & 0.778 & 10.46 & 10.46 & 7.9 & 5.04 & 3.16 & 0.302 & 0.183 & 0.158 \\
\hline fabp4 & 0.747 & 50.97 & 31.86 & 18.05 & 5.84 & 2.55 & 0.46 & 0.509 & 0.803 \\
\hline fak1 & 0.853 & 42.39 & 24.77 & 20 & 8.38 & 3.8 & 0.583 & 0.508 & 0.734 \\
\hline fgfr1 & 0.753 & 2.89 & 2.89 & 2.15 & 2.88 & 2.88 & 0.158 & 0.053 & 0.043 \\
\hline fkbla & 0.638 & 5.33 & 6.32 & 4.51 & 2.81 & 1.8 & 0.178 & 0.107 & 0.068 \\
\hline fnta & 0.832 & 14.18 & 13.17 & 12.83 & 7.92 & 3.82 & 0.443 & 0.233 & 0.159 \\
\hline fpps & 0.967 & 64.77 & 67.73 & 40.64 & 11.58 & 4.75 & 0.842 & 0.718 & 0.531 \\
\hline gcr & 0.475 & 3.11 & 3.5 & 3.68 & 1.89 & 1.09 & 0.119 & 0.074 & 0.053 \\
\hline $\mathrm{glcm}$ & 0.732 & 33.8 & 25.61 & 14.83 & 5.33 & 3.15 & 0.392 & 0.356 & 0.511 \\
\hline gria2 & 0.764 & 51.8 & 32.22 & 18.32 & 6.01 & 2.79 & 0.447 & 0.448 & 0.677 \\
\hline grik1 & 0.823 & 53.81 & 35.87 & 21.76 & 7.18 & 3.57 & 0.542 & 0.53 & 0.759 \\
\hline hdac2 & 0.798 & 26.15 & 19.97 & 14.84 & 7.36 & 3.46 & 0.472 & 0.365 & 0.45 \\
\hline hdac8 & 0.9 & 41.24 & 37.11 & 32.99 & 10.3 & 4.29 & 0.742 & 0.656 & 0.664 \\
\hline hivpr & 0.66 & 6.36 & 4.11 & 3.27 & 2.64 & 1.87 & 0.151 & 0.08 & 0.094 \\
\hline hivrt & 0.751 & 24.86 & 18.94 & 13.61 & 5.47 & 2.75 & 0.382 & 0.335 & 0.453 \\
\hline hmdh & 0.881 & 52.42 & 52.42 & 30.04 & 8.9 & 3.97 & 0.705 & 0.766 & 0.972 \\
\hline hs90a & 0.705 & 22.43 & 21.75 & 12.46 & 5.39 & 2.84 & 0.378 & 0.331 & 0.444 \\
\hline hxk4 & 0.528 & 41.2 & 20.6 & 12.47 & 3.26 & 1.3 & 0.286 & 0.378 & 0.725 \\
\hline igflr & 0.843 & 54.27 & 35.95 & 21.25 & 7.77 & 3.65 & 0.562 & 0.54 & 0.781 \\
\hline inha & 0.7 & 4.54 & 2.37 & 2.32 & 2.04 & 1.86 & 0.118 & 0.061 & 0.099 \\
\hline jak2 & 0.906 & 57.95 & 36.45 & 24.77 & 9.11 & 4.25 & 0.636 & 0.602 & 0.829 \\
\hline kif11 & 0.721 & 44.59 & 30.01 & 17.71 & 6.14 & 2.97 & 0.448 & 0.463 & 0.684 \\
\hline kit & 0.662 & 7.24 & 9.05 & 6.63 & 3.61 & 2.47 & 0.23 & 0.15 & 0.168 \\
\hline kith & 0.767 & 50.98 & 50.98 & 31.64 & 8.57 & 3.42 & 0.701 & 0.792 & 0.979 \\
\hline $\mathrm{kpcb}$ & 0.871 & 66.29 & 58 & 32.01 & 9.12 & 4.06 & 0.725 & 0.765 & 0.952 \\
\hline lck & 0.801 & 34.27 & 22.37 & 14.76 & 6.04 & 3.27 & 0.42 & 0.368 & 0.541 \\
\hline lkha4 & 0.869 & 17.58 & 14.65 & 8.79 & 5.99 & 4.06 & 0.358 & 0.237 & 0.308 \\
\hline mapk2 & 0.952 & 61.85 & 59.86 & 38.1 & 10.39 & 4.51 & 0.839 & 0.863 & 0.974 \\
\hline met & 0.9 & 68.67 & 56.63 & 31.02 & 9.26 & 4.1 & 0.711 & 0.738 & 0.935 \\
\hline $\mathrm{mk} 01$ & 0.692 & 25.47 & 28.01 & 16.37 & 5.54 & 2.66 & 0.405 & 0.389 & 0.401 \\
\hline $\mathrm{mk} 10$ & 0.775 & 11.37 & 8.66 & 5.29 & 4.09 & 2.64 & 0.246 & 0.151 & 0.192 \\
\hline $\mathrm{mk} 14$ & 0.731 & 16.6 & 11.93 & 8.47 & 4.11 & 2.73 & 0.271 & 0.206 & 0.296 \\
\hline mmp 13 & 0.827 & 39.94 & 27.08 & 16.01 & 5.88 & 3.36 & 0.429 & 0.409 & 0.624 \\
\hline
\end{tabular}




\begin{tabular}{|c|c|c|c|c|c|c|c|c|c|} 
nos1 & 0.704 & 7.95 & 9.05 & 6 & 3.5 & 2.2 & 0.208 & 0.121 & 0.109 \\
\hline nram & 0.886 & 60.1 & 39.77 & 24.98 & 9.05 & 4.08 & 0.646 & 0.624 & 0.847 \\
\hline pa2ga & 0.756 & 48.9 & 30.56 & 17.15 & 5.93 & 3.23 & 0.463 & 0.505 & 0.821 \\
\hline parp1 & 0.843 & 33.79 & 23.26 & 16.23 & 7.23 & 3.71 & 0.485 & 0.412 & 0.589 \\
\hline pde5a & 0.799 & 38.07 & 22.87 & 14.83 & 6.09 & 3.14 & 0.418 & 0.371 & 0.572 \\
\hline pgh2 & 0.743 & 33.58 & 21.39 & 13.26 & 5.24 & 2.81 & 0.383 & 0.376 & 0.607 \\
\hline plk1 & 0.89 & 47.93 & 33.66 & 22.44 & 8.3 & 4.06 & 0.6 & 0.538 & 0.714 \\
\hline pnph & 0.932 & 66.51 & 54.97 & 33.02 & 9.1 & 4.27 & 0.72 & 0.748 & 0.921 \\
\hline ppara & 0.799 & 30.44 & 20.94 & 13.82 & 5.86 & 3.26 & 0.419 & 0.383 & 0.59 \\
\hline ppard & 0.745 & 8.37 & 7.06 & 5.42 & 3.91 & 2.88 & 0.242 & 0.146 & 0.165 \\
\hline pparg & 0.724 & 20.6 & 14.69 & 10.53 & 4.78 & 2.58 & 0.323 & 0.274 & 0.411 \\
\hline prgr & 0.54 & 16.31 & 11.28 & 7.5 & 3.03 & 1.59 & 0.212 & 0.206 & 0.353 \\
\hline ptn1 & 0.795 & 4.6 & 2.3 & 3.47 & 5.09 & 3.15 & 0.259 & 0.096 & 0.124 \\
\hline pur2 & 0.997 & 54.84 & 54.84 & 45.87 & 12.27 & 5 & 0.969 & 0.969 & 0.998 \\
\hline pyrd & 0.863 & 53.69 & 36.69 & 22.54 & 7.33 & 3.47 & 0.569 & 0.583 & 0.831 \\
\hline reni & 0.72 & 42.66 & 23.9 & 13.48 & 5.29 & 2.69 & 0.375 & 0.37 & 0.623 \\
\hline rock1 & 0.857 & 29.98 & 28.98 & 21.48 & 8.37 & 3.9 & 0.561 & 0.448 & 0.405 \\
\hline sahh & 0.981 & 55.76 & 55.76 & 47.8 & 12.1 & 4.92 & 0.966 & 0.977 & 0.999 \\
\hline src & 0.745 & 17.6 & 13.01 & 9.29 & 4.31 & 2.52 & 0.28 & 0.218 & 0.307 \\
\hline tgfr1 & 0.943 & 58.84 & 52.05 & 34.13 & 10.16 & 4.36 & 0.772 & 0.759 & 0.889 \\
\hline thb & 0.86 & 57.8 & 37.09 & 20.36 & 7.04 & 3.69 & 0.53 & 0.519 & 0.742 \\
\hline thrb & 0.828 & 46.94 & 31.3 & 18.58 & 7.39 & 3.61 & 0.526 & 0.502 & 0.748 \\
\hline try1 & 0.921 & 58.36 & 49.63 & 32.25 & 9.77 & 4.29 & 0.756 & 0.763 & 0.94 \\
\hline tryb1 & 0.718 & 36.42 & 22.93 & 13.49 & 4.81 & 2.47 & 0.374 & 0.402 & 0.692 \\
\hline tysy & 0.825 & 31.37 & 23.07 & 18.78 & 7.11 & 3.72 & 0.483 & 0.411 & 0.529 \\
\hline urok & 0.923 & 61.75 & 58.66 & 34.58 & 10.03 & 4.32 & 0.779 & 0.806 & 0.967 \\
\hline vgfr2 & 0.794 & 23.9 & 15.67 & 11.24 & 5.14 & 3.06 & 0.348 & 0.284 & 0.422 \\
\hline wee1 & 0.99 & 61.27 & 61.27 & 48.53 & 12.38 & 4.95 & 0.984 & 0.985 & 0.999 \\
\hline xiap & 0.933 & 52.44 & 52.44 & 44.45 & 11.24 & 4.5 & 0.911 & 0.948 & 0.998 \\
\hline
\end{tabular}

B

\begin{tabular}{|c|c|c|c|c|c|c|c|c|c|}
\hline Complex & AUC & $\begin{array}{c}\text { EF } \\
0.5 \%\end{array}$ & $\begin{array}{c}\text { EF } \\
1 \%\end{array}$ & $\begin{array}{c}\text { EF } \\
2 \%\end{array}$ & $\begin{array}{c}\text { EF } \\
8 \%\end{array}$ & $\begin{array}{c}\text { EF } \\
20 \%\end{array}$ & $\begin{array}{c}\text { BEDROC } \\
\alpha=20\end{array}$ & $\begin{array}{c}\text { BEDROC } \\
\alpha=80.5\end{array}$ & $\begin{array}{c}\text { BEDROC } \\
\alpha=321.0\end{array}$ \\
\hline aa2ar & 0.887 & 53.69 & 39.54 & 22.51 & 9.06 & 4.04 & 0.618 & 0.567 & 0.758 \\
\hline abl1 & 0.737 & 33.84 & 20.38 & 12.34 & 5.36 & 2.83 & 0.371 & 0.348 & 0.585 \\
\hline ace & 0.787 & 26.81 & 17.39 & 10.82 & 5.85 & 3.33 & 0.371 & 0.291 & 0.416 \\
\hline aces & 0.594 & 3.98 & 2.87 & 3.09 & 2.35 & 1.80 & 0.131 & 0.068 & 0.069 \\
\hline ada & 0.904 & 55.33 & 44.42 & 28.99 & 9.01 & 4.30 & 0.686 & 0.689 & 0.878 \\
\hline ada17 & 0.718 & 7.13 & 6.40 & 4.51 & 3.62 & 2.73 & 0.207 & 0.105 & 0.095 \\
\hline adrb1 & 0.822 & 42.33 & 24.68 & 18.00 & 7.08 & 3.52 & 0.491 & 0.434 & 0.632 \\
\hline adrb2 & 0.678 & 10.40 & 6.94 & 4.33 & 3.30 & 2.40 & 0.205 & 0.122 & 0.171 \\
\hline akt1 & 0.731 & 18.33 & 14.00 & 9.05 & 4.86 & 2.95 & 0.318 & 0.242 & 0.345 \\
\hline
\end{tabular}




\begin{tabular}{|c|c|c|c|c|c|c|c|c|c|}
\hline akt2 & 0.657 & 22.25 & 11.98 & 6.85 & 3.10 & 2.35 & 0.231 & 0.213 & 0.417 \\
\hline aldr & 0.766 & 43.76 & 32.23 & 17.91 & 6.36 & 3.14 & 0.475 & 0.489 & 0.758 \\
\hline ampc & 0.849 & 30.00 & 22.76 & 18.62 & 6.78 & 3.65 & 0.497 & 0.426 & 0.521 \\
\hline andr & 0.634 & 14.93 & 8.21 & 5.41 & 2.94 & 1.90 & 0.201 & 0.171 & 0.343 \\
\hline bace1 & 0.612 & 4.23 & 2.47 & 2.12 & 1.94 & 1.45 & 0.113 & 0.054 & 0.067 \\
\hline braf & 0.784 & 55.78 & 30.90 & 17.75 & 5.59 & 3.12 & 0.449 & 0.474 & 0.764 \\
\hline cah2 & 0.577 & 1.22 & 1.02 & 1.22 & 0.94 & 1.40 & 0.067 & 0.026 & 0.023 \\
\hline casp3 & 0.681 & 11.14 & 8.53 & 8.03 & 4.90 & 2.69 & 0.299 & 0.188 & 0.184 \\
\hline $\mathrm{cdk} 2$ & 0.780 & 20.18 & 15.19 & 10.87 & 5.17 & 3.16 & 0.351 & 0.266 & 0.314 \\
\hline comt & 0.955 & 79.88 & 38.91 & 25.54 & 10.67 & 4.88 & 0.706 & 0.581 & 0.765 \\
\hline cp2c9 & 0.684 & 4.98 & 3.32 & 3.76 & 2.29 & 2.29 & 0.146 & 0.073 & 0.059 \\
\hline cp3a4 & 0.732 & 14.08 & 11.14 & 8.84 & 4.85 & 2.71 & 0.296 & 0.195 & 0.236 \\
\hline csflr & 0.791 & 23.92 & 18.69 & 12.66 & 6.55 & 3.04 & 0.400 & 0.279 & 0.284 \\
\hline cxcr4 & 0.775 & 15.20 & 12.67 & 13.73 & 7.18 & 3.75 & 0.427 & 0.237 & 0.214 \\
\hline def & 0.870 & 47.04 & 36.26 & 20.09 & 7.35 & 3.77 & 0.545 & 0.554 & 0.811 \\
\hline dhil & 0.613 & 6.08 & 4.84 & 3.64 & 2.39 & 1.92 & 0.145 & 0.085 & 0.092 \\
\hline dpp4 & 0.631 & 28.60 & 15.43 & 8.74 & 3.85 & 2.18 & 0.259 & 0.232 & 0.388 \\
\hline drd3 & 0.556 & 3.34 & 2.71 & 2.39 & 1.77 & 1.46 & 0.102 & 0.053 & 0.067 \\
\hline dyr & 0.892 & 48.48 & 36.79 & 24.24 & 8.87 & 4.18 & 0.629 & 0.542 & 0.666 \\
\hline egfr & 0.798 & 26.95 & 16.98 & 11.92 & 5.73 & 3.30 & 0.374 & 0.294 & 0.445 \\
\hline esr1 & 0.842 & 54.39 & 44.71 & 25.17 & 8.72 & 3.89 & 0.649 & 0.668 & 0.913 \\
\hline esr2 & 0.826 & 52.16 & 46.13 & 25.73 & 7.60 & 3.86 & 0.608 & 0.661 & 0.896 \\
\hline fa10 & 0.636 & 7.83 & 6.34 & 5.23 & 3.24 & 2.15 & 0.198 & 0.124 & 0.143 \\
\hline fa7 & 0.551 & 1.74 & 1.74 & 1.32 & 1.42 & 1.45 & 0.088 & 0.039 & 0.051 \\
\hline fabp4 & 0.729 & 59.47 & 36.11 & 18.05 & 5.58 & 2.45 & 0.443 & 0.521 & 0.852 \\
\hline fak1 & 0.893 & 52.48 & 51.47 & 32.00 & 9.50 & 4.05 & 0.748 & 0.786 & 0.951 \\
\hline fgfr1 & 0.739 & 14.43 & 13.71 & 10.40 & 4.95 & 3.02 & 0.313 & 0.222 & 0.228 \\
\hline fkbla & 0.522 & 5.33 & 4.51 & 4.06 & 1.69 & 1.67 & 0.136 & 0.096 & 0.060 \\
\hline fnta & 0.829 & 53.02 & 35.80 & 22.97 & 8.00 & 3.84 & 0.568 & 0.490 & 0.596 \\
\hline fpps & 0.992 & 97.15 & 76.35 & 44.95 & 12.35 & 5.00 & 0.934 & 0.870 & 0.870 \\
\hline gcr & 0.709 & 10.88 & 7.77 & 6.00 & 3.97 & 2.67 & 0.245 & 0.152 & 0.187 \\
\hline glcm & 0.829 & 52.58 & 36.59 & 24.09 & 7.41 & 3.61 & 0.564 & 0.547 & 0.682 \\
\hline gria2 & 0.747 & 61.91 & 40.43 & 22.74 & 6.80 & 3.20 & 0.524 & 0.545 & 0.768 \\
\hline grik1 & 0.759 & 25.91 & 23.91 & 12.86 & 5.45 & 2.97 & 0.370 & 0.315 & 0.378 \\
\hline hdac2 & 0.564 & 17.44 & 10.25 & 5.94 & 2.50 & 1.59 & 0.183 & 0.182 & 0.367 \\
\hline hdac8 & 0.783 & 18.85 & 15.32 & 12.96 & 6.77 & 3.35 & 0.431 & 0.309 & 0.356 \\
\hline hivpr & 0.613 & 2.99 & 2.99 & 3.46 & 2.55 & 1.77 & 0.147 & 0.067 & 0.044 \\
\hline hivrt & 0.682 & 6.51 & 5.62 & 5.03 & 3.14 & 2.31 & 0.194 & 0.117 & 0.133 \\
\hline hmdh & 0.886 & 52.42 & 49.47 & 30.04 & 8.45 & 3.94 & 0.687 & 0.759 & 0.964 \\
\hline hs90a & 0.735 & 17.95 & 17.17 & 13.60 & 5.39 & 2.95 & 0.389 & 0.314 & 0.289 \\
\hline hxk4 & 0.875 & 45.54 & 31.44 & 21.68 & 7.47 & 3.69 & 0.578 & 0.570 & 0.818 \\
\hline igflr & 0.808 & 32.56 & 24.42 & 17.88 & 5.91 & 3.18 & 0.444 & 0.411 & 0.608 \\
\hline
\end{tabular}




\begin{tabular}{|c|c|c|c|c|c|c|c|c|c|}
\hline inha & 0.532 & 9.08 & 4.74 & 3.48 & 2.04 & 1.28 & 0.137 & 0.107 & 0.159 \\
\hline jak2 & 0.865 & 44.87 & 28.04 & 17.29 & 7.13 & 4.02 & 0.511 & 0.454 & 0.619 \\
\hline kif11 & 0.626 & 5.15 & 3.43 & 4.32 & 3.34 & 2.07 & 0.185 & 0.093 & 0.095 \\
\hline kit & 0.654 & 12.06 & 7.24 & 6.03 & 3.24 & 2.05 & 0.205 & 0.138 & 0.189 \\
\hline kith & 0.895 & 50.98 & 50.98 & 35.16 & 9.23 & 3.86 & 0.768 & 0.847 & 0.988 \\
\hline kpcb & 0.883 & 66.29 & 57.25 & 33.14 & 9.59 & 4.21 & 0.753 & 0.779 & 0.949 \\
\hline lck & 0.743 & 18.56 & 12.38 & 9.40 & 4.55 & 2.87 & 0.306 & 0.224 & 0.299 \\
\hline lkha4 & 0.775 & 3.52 & 5.27 & 3.52 & 4.02 & 3.54 & 0.231 & 0.094 & 0.067 \\
\hline mapk2 & 0.907 & 45.89 & 32.92 & 25.24 & 9.77 & 4.21 & 0.676 & 0.569 & 0.628 \\
\hline met & 0.863 & 61.45 & 50.60 & 31.02 & 9.19 & 3.89 & 0.702 & 0.707 & 0.864 \\
\hline $\mathrm{mk} 01$ & 0.649 & 58.57 & 33.10 & 17.00 & 4.75 & 2.53 & 0.404 & 0.497 & 0.837 \\
\hline mk10 & 0.651 & 3.79 & 1.92 & 0.96 & 2.16 & 1.87 & 0.106 & 0.036 & 0.058 \\
\hline mk14 & 0.678 & 15.22 & 10.37 & 7.18 & 3.48 & 2.26 & 0.232 & 0.177 & 0.244 \\
\hline mmp 13 & 0.794 & 32.23 & 23.41 & 14.26 & 5.99 & 3.35 & 0.414 & 0.361 & 0.533 \\
\hline nos1 & 0.701 & 9.94 & 8.05 & 5.50 & 3.50 & 2.75 & 0.204 & 0.114 & 0.136 \\
\hline nram & 0.891 & 31.09 & 22.43 & 19.89 & 8.16 & 4.34 & 0.543 & 0.413 & 0.449 \\
\hline pa2ga & 0.677 & 24.45 & 14.26 & 10.09 & 3.91 & 2.42 & 0.292 & 0.268 & 0.369 \\
\hline parp1 & 0.844 & 42.04 & 28.58 & 18.99 & 7.23 & 3.77 & 0.524 & 0.485 & 0.706 \\
\hline pde $5 a$ & 0.750 & 38.57 & 23.88 & 13.82 & 5.50 & 2.95 & 0.383 & 0.360 & 0.589 \\
\hline pgh2 & 0.795 & 37.73 & 24.38 & 15.91 & 6.42 & 3.31 & 0.463 & 0.435 & 0.665 \\
\hline plk1 & 0.805 & 9.22 & 7.48 & 9.35 & 5.84 & 3.50 & 0.343 & 0.182 & 0.166 \\
\hline pnph & 0.910 & 66.51 & 55.93 & 33.99 & 10.08 & 4.18 & 0.760 & 0.764 & 0.911 \\
\hline ppara & 0.817 & 36.85 & 29.53 & 19.46 & 7.27 & 3.59 & 0.524 & 0.508 & 0.711 \\
\hline ppard & 0.658 & 5.86 & 3.74 & 2.92 & 2.55 & 2.10 & 0.153 & 0.084 & 0.129 \\
\hline pparg & 0.742 & 28.02 & 17.99 & 11.77 & 4.75 & 2.83 & 0.355 & 0.336 & 0.541 \\
\hline prgr & 0.629 & 19.03 & 11.97 & 7.33 & 3.07 & 1.89 & 0.222 & 0.212 & 0.386 \\
\hline ptn1 & 0.718 & 3.06 & 1.53 & 1.54 & 2.02 & 2.42 & 0.131 & 0.048 & 0.033 \\
\hline pur2 & 0.999 & 54.84 & 54.84 & 47.86 & 12.52 & 5.00 & 0.992 & 0.987 & 0.999 \\
\hline pyrd & 0.738 & 59.05 & 40.26 & 22.99 & 6.54 & 2.97 & 0.538 & 0.609 & 0.882 \\
\hline reni & 0.736 & 21.33 & 15.29 & 12.03 & 5.04 & 2.84 & 0.342 & 0.274 & 0.376 \\
\hline rock1 & 0.829 & 17.99 & 14.99 & 14.99 & 6.37 & 3.55 & 0.428 & 0.298 & 0.271 \\
\hline sahh & 0.998 & 55.76 & 55.76 & 47.00 & 12.50 & 5.00 & 0.971 & 0.961 & 0.995 \\
\hline src & 0.745 & 11.10 & 8.61 & 6.42 & 4.50 & 2.79 & 0.259 & 0.154 & 0.200 \\
\hline $\operatorname{tgfr} 1$ & 0.923 & 43.76 & 33.19 & 25.50 & 9.50 & 4.40 & 0.656 & 0.562 & 0.677 \\
\hline thb & 0.888 & 52.02 & 35.14 & 23.27 & 8.62 & 4.18 & 0.592 & 0.526 & 0.688 \\
\hline thrb & 0.729 & 15.65 & 12.82 & 9.02 & 4.54 & 2.67 & 0.297 & 0.226 & 0.299 \\
\hline try1 & 0.804 & 20.49 & 16.99 & 11.83 & 6.00 & 3.40 & 0.396 & 0.297 & 0.391 \\
\hline tryb1 & 0.577 & 13.49 & 10.12 & 5.73 & 2.70 & 1.66 & 0.195 & 0.179 & 0.342 \\
\hline tysy & 0.813 & 38.75 & 30.45 & 19.24 & 6.77 & 3.49 & 0.501 & 0.469 & 0.648 \\
\hline urok & 0.884 & 41.99 & 36.43 & 28.09 & 8.49 & 4.07 & 0.639 & 0.595 & 0.678 \\
\hline vgfr2 & 0.740 & 15.61 & 10.53 & 8.55 & 3.97 & 2.64 & 0.269 & 0.199 & 0.263 \\
\hline wee1 & 0.997 & 61.27 & 61.27 & 44.61 & 12.38 & 5.00 & 0.966 & 0.959 & 0.996 \\
\hline
\end{tabular}




\begin{tabular}{|l|l|l|l|l|l|l|l|l|l|} 
xiap & 0.919 & 36.30 & 35.30 & 24.97 & 10.99 & 4.45 & 0.721 & 0.617 & 0.720 \\
\hline
\end{tabular}

C

\begin{tabular}{|c|c|c|c|c|c|c|c|c|c|}
\hline Complex & AUC & $\begin{array}{c}\text { EF } \\
0.5 \% \\
\end{array}$ & $\begin{array}{l}\mathrm{EF} \\
1 \% \\
\end{array}$ & $\begin{array}{l}\mathrm{EF} \\
2 \% \\
\end{array}$ & $\begin{array}{l}\text { EF } \\
8 \% \\
\end{array}$ & $\begin{array}{c}\mathrm{EF} \\
20 \% \\
\end{array}$ & $\begin{array}{c}\text { BEDROC } \\
\alpha=20 \\
\end{array}$ & $\begin{array}{c}\text { BEDROC } \\
\alpha=80.5\end{array}$ & $\begin{array}{c}\text { BEDROC } \\
\alpha=321.0\end{array}$ \\
\hline aa2ar & 0.734 & 3.75 & 4.79 & 4.69 & 3.13 & 2.19 & 0.178 & 0.089 & 0.066 \\
\hline abl1 & 0.577 & 17.46 & 13.77 & 9.32 & 3.30 & 1.59 & 0.241 & 0.230 & 0.334 \\
\hline ace & 0.596 & 8.47 & 5.32 & 3.37 & 1.73 & 1.67 & 0.131 & 0.094 & 0.138 \\
\hline aces & 0.508 & 4.43 & 2.87 & 2.21 & 1.32 & 1.19 & 0.087 & 0.056 & 0.077 \\
\hline ada & 0.751 & 14.63 & 14.67 & 11.08 & 5.73 & 3.10 & 0.357 & 0.236 & 0.211 \\
\hline ada17 & 0.653 & 2.13 & 3.25 & 2.15 & 2.29 & 2.21 & 0.144 & 0.052 & 0.032 \\
\hline adrb1 & 0.694 & 7.33 & 6.07 & 6.07 & 3.34 & 2.37 & 0.214 & 0.125 & 0.097 \\
\hline adrb2 & 0.626 & 5.20 & 3.47 & 2.38 & 1.62 & 1.47 & 0.101 & 0.058 & 0.076 \\
\hline akt1 & 0.653 & 8.82 & 6.15 & 6.32 & 3.45 & 2.22 & 0.221 & 0.142 & 0.148 \\
\hline akt2 & 0.624 & 6.85 & 4.28 & 2.57 & 1.60 & 1.54 & 0.110 & 0.075 & 0.137 \\
\hline aldr & 0.684 & 28.76 & 16.43 & 9.74 & 4.16 & 2.45 & 0.298 & 0.292 & 0.531 \\
\hline ampc & 0.676 & 0.00 & 0.00 & 2.07 & 2.61 & 2.29 & 0.131 & 0.030 & 0.002 \\
\hline andr & 0.495 & 7.47 & 4.48 & 2.43 & 1.26 & 1.06 & 0.086 & 0.073 & 0.124 \\
\hline bace1 & 0.697 & 8.46 & 7.05 & 6.54 & 2.91 & 2.14 & 0.200 & 0.137 & 0.149 \\
\hline braf & 0.797 & 17.27 & 13.81 & 12.16 & 6.00 & 3.26 & 0.367 & 0.256 & 0.283 \\
\hline cah2 & 0.919 & 37.00 & 32.13 & 26.23 & 10.14 & 4.38 & 0.690 & 0.552 & 0.558 \\
\hline casp3 & 0.727 & 9.12 & 5.52 & 4.27 & 3.14 & 2.54 & 0.197 & 0.118 & 0.156 \\
\hline cdk2 & 0.819 & 19.34 & 16.04 & 13.19 & 6.09 & 3.42 & 0.399 & 0.298 & 0.359 \\
\hline comt & 0.906 & 0.00 & 0.00 & 1.22 & 4.27 & 4.88 & 0.207 & 0.014 & 0.000 \\
\hline cp2c9 & 0.558 & 4.98 & 3.32 & 4.17 & 1.98 & 1.37 & 0.126 & 0.075 & 0.065 \\
\hline cp3a4 & 0.673 & 5.87 & 5.28 & 4.71 & 2.87 & 2.00 & 0.170 & 0.095 & 0.092 \\
\hline csflr & 0.757 & 27.51 & 27.73 & 16.28 & 5.72 & 3.01 & 0.415 & 0.356 & 0.393 \\
\hline cxcr4 & 0.717 & 20.27 & 12.67 & 6.24 & 4.06 & 3.25 & 0.246 & 0.173 & 0.282 \\
\hline def & 0.457 & 1.96 & 1.96 & 0.98 & 0.37 & 0.69 & 0.034 & 0.025 & 0.025 \\
\hline dhil & 0.503 & 0.00 & 0.00 & 0.91 & 1.10 & 0.97 & 0.055 & 0.013 & 0.001 \\
\hline dpp4 & 0.709 & 9.78 & 7.71 & 6.77 & 3.57 & 2.42 & 0.225 & 0.134 & 0.119 \\
\hline drd3 & 0.642 & 2.51 & 3.75 & 3.96 & 2.42 & 1.97 & 0.145 & 0.068 & 0.032 \\
\hline dyr & 0.884 & 65.79 & 49.34 & 31.60 & 9.04 & 3.96 & 0.692 & 0.680 & 0.836 \\
\hline egfr & 0.749 & 27.69 & 16.98 & 11.55 & 5.20 & 2.89 & 0.345 & 0.290 & 0.450 \\
\hline esr1 & 0.751 & 40.79 & 27.46 & 15.39 & 5.13 & 2.86 & 0.405 & 0.437 & 0.713 \\
\hline esr2 & 0.803 & 39.12 & 25.93 & 15.93 & 6.51 & 3.09 & 0.457 & 0.438 & 0.674 \\
\hline fa10 & 0.690 & 11.55 & 7.08 & 4.95 & 3.17 & 2.31 & 0.204 & 0.142 & 0.217 \\
\hline fa7 & 0.883 & 20.91 & 13.94 & 14.93 & 7.78 & 4.17 & 0.487 & 0.335 & 0.381 \\
\hline fabp4 & 0.667 & 8.50 & 4.25 & 4.25 & 1.86 & 1.81 & 0.137 & 0.104 & 0.181 \\
\hline fak1 & 0.887 & 22.20 & 21.80 & 16.50 & 8.38 & 3.90 & 0.550 & 0.415 & 0.446 \\
\hline fgfr1 & 0.743 & 5.77 & 6.49 & 6.46 & 5.04 & 3.02 & 0.285 & 0.138 & 0.072 \\
\hline fkbla & 0.645 & 0.00 & 0.90 & 0.45 & 1.80 & 1.67 & 0.080 & 0.015 & 0.004 \\
\hline
\end{tabular}




\begin{tabular}{|c|c|c|c|c|c|c|c|c|c|}
\hline fnta & 0.704 & 5.40 & 5.07 & 4.90 & 3.27 & 2.28 & 0.183 & 0.090 & 0.074 \\
\hline fpps & 0.788 & 17.44 & 13.55 & 16.63 & 6.33 & 3.02 & 0.402 & 0.248 & 0.133 \\
\hline gcr & 0.464 & 3.11 & 1.94 & 1.36 & 1.11 & 0.91 & 0.071 & 0.040 & 0.050 \\
\hline glcm & 0.660 & 26.29 & 20.12 & 13.90 & 5.10 & 2.59 & 0.359 & 0.309 & 0.414 \\
\hline gria2 & 0.719 & 32.85 & 20.85 & 13.27 & 5.22 & 2.69 & 0.360 & 0.319 & 0.483 \\
\hline grik1 & 0.778 & 39.86 & 25.91 & 16.32 & 5.82 & 3.02 & 0.415 & 0.402 & 0.616 \\
\hline hdac2 & 0.757 & 22.88 & 21.05 & 13.49 & 5.27 & 2.97 & 0.379 & 0.328 & 0.358 \\
\hline hdac8 & 0.763 & 14.14 & 14.14 & 11.49 & 5.30 & 3.15 & 0.349 & 0.238 & 0.210 \\
\hline hivpr & 0.660 & 8.60 & 6.54 & 5.52 & 3.18 & 2.11 & 0.192 & 0.119 & 0.134 \\
\hline hivrt & 0.631 & 10.06 & 7.10 & 5.18 & 2.70 & 1.86 & 0.176 & 0.129 & 0.171 \\
\hline hmdh & 0.835 & 52.42 & 41.23 & 23.56 & 7.43 & 3.59 & 0.594 & 0.649 & 0.913 \\
\hline hs90a & 0.617 & 2.24 & 4.58 & 3.97 & 2.56 & 1.93 & 0.149 & 0.074 & 0.040 \\
\hline hxk4 & 0.560 & 28.19 & 16.26 & 8.67 & 3.26 & 1.41 & 0.249 & 0.287 & 0.553 \\
\hline igflr & 0.671 & 31.20 & 16.96 & 10.80 & 3.88 & 2.26 & 0.288 & 0.293 & 0.524 \\
\hline inha & 0.715 & 0.00 & 0.00 & 0.00 & 2.62 & 1.98 & 0.093 & 0.004 & 0.000 \\
\hline jak2 & 0.826 & 33.65 & 27.11 & 18.69 & 6.31 & 3.36 & 0.478 & 0.438 & 0.555 \\
\hline kif11 & 0.716 & 17.15 & 12.86 & 8.20 & 4.63 & 2.46 & 0.292 & 0.209 & 0.239 \\
\hline kit & 0.623 & 2.41 & 3.02 & 3.32 & 2.33 & 1.93 & 0.142 & 0.063 & 0.032 \\
\hline kith & 0.762 & 10.20 & 5.27 & 5.27 & 2.86 & 3.07 & 0.217 & 0.145 & 0.225 \\
\hline kpcb & 0.822 & 63.27 & 48.96 & 26.36 & 8.18 & 3.68 & 0.625 & 0.652 & 0.876 \\
\hline lck & 0.773 & 31.42 & 19.99 & 13.21 & 5.27 & 2.94 & 0.369 & 0.323 & 0.467 \\
\hline lkha4 & 0.656 & 7.03 & 5.27 & 3.52 & 3.36 & 2.66 & 0.197 & 0.102 & 0.105 \\
\hline mapk2 & 0.808 & 41.90 & 28.93 & 17.32 & 6.68 & 3.42 & 0.490 & 0.466 & 0.695 \\
\hline met & 0.846 & 57.83 & 36.75 & 21.99 & 7.30 & 3.64 & 0.537 & 0.527 & 0.757 \\
\hline $\mathrm{mk} 01$ & 0.691 & 5.09 & 6.37 & 6.30 & 4.27 & 2.53 & 0.247 & 0.137 & 0.085 \\
\hline $\mathrm{mk} 10$ & 0.792 & 20.85 & 12.51 & 7.21 & 4.57 & 2.88 & 0.286 & 0.212 & 0.297 \\
\hline $\mathrm{mk} 14$ & 0.696 & 13.83 & 8.99 & 6.92 & 3.53 & 2.32 & 0.229 & 0.170 & 0.242 \\
\hline mmp13 & 0.755 & 19.62 & 14.33 & 9.62 & 4.59 & 3.00 & 0.301 & 0.228 & 0.290 \\
\hline nos1 & 0.569 & 3.97 & 3.02 & 1.50 & 2.37 & 1.50 & 0.122 & 0.048 & 0.040 \\
\hline nram & 0.715 & 26.94 & 19.38 & 13.26 & 5.48 & 2.65 & 0.378 & 0.326 & 0.478 \\
\hline pa2ga & 0.849 & 40.75 & 32.60 & 19.17 & 7.19 & 3.74 & 0.526 & 0.512 & 0.730 \\
\hline parp1 & 0.814 & 18.86 & 18.53 & 14.17 & 6.20 & 3.46 & 0.426 & 0.325 & 0.328 \\
\hline pde $5 a$ & 0.697 & 5.51 & 5.28 & 3.77 & 2.64 & 2.41 & 0.164 & 0.086 & 0.100 \\
\hline pgh2 & 0.745 & 19.78 & 13.11 & 9.11 & 3.89 & 2.72 & 0.277 & 0.245 & 0.410 \\
\hline plk1 & 0.836 & 44.24 & 32.73 & 20.11 & 7.95 & 3.50 & 0.538 & 0.503 & 0.699 \\
\hline pnph & 0.743 & 52.82 & 39.54 & 22.82 & 6.68 & 3.01 & 0.518 & 0.538 & 0.726 \\
\hline ppara & 0.807 & 18.16 & 12.35 & 10.60 & 5.50 & 3.19 & 0.349 & 0.261 & 0.366 \\
\hline ppard & 0.746 & 8.37 & 6.23 & 7.09 & 4.06 & 2.60 & 0.251 & 0.156 & 0.148 \\
\hline pparg & 0.764 & 16.89 & 14.69 & 11.77 & 5.30 & 3.03 & 0.365 & 0.287 & 0.360 \\
\hline prgr & 0.575 & 8.15 & 9.57 & 5.62 & 2.82 & 1.66 & 0.194 & 0.152 & 0.181 \\
\hline ptn1 & 0.789 & 6.13 & 5.36 & 6.17 & 5.48 & 3.23 & 0.299 & 0.134 & 0.095 \\
\hline pur2 & 0.984 & 54.84 & 54.84 & 41.88 & 11.52 & 4.80 & 0.914 & 0.926 & 0.992 \\
\hline
\end{tabular}




\begin{tabular}{|c|c|c|c|c|c|c|c|c|c|} 
pyrd & 0.829 & 28.63 & 22.37 & 14.43 & 7.10 & 3.78 & 0.450 & 0.364 & 0.452 \\
\hline reni & 0.667 & 31.02 & 19.12 & 12.51 & 4.32 & 2.50 & 0.324 & 0.304 & 0.476 \\
\hline rock1 & 0.769 & 25.98 & 14.99 & 9.49 & 4.75 & 2.90 & 0.326 & 0.268 & 0.402 \\
\hline sahh & 0.921 & 52.66 & 49.39 & 32.66 & 9.72 & 4.28 & 0.755 & 0.776 & 0.937 \\
\hline src & 0.747 & 14.93 & 10.14 & 7.28 & 3.28 & 2.38 & 0.226 & 0.175 & 0.249 \\
\hline tgfr1 & 0.881 & 52.81 & 43.00 & 30.00 & 9.40 & 4.21 & 0.687 & 0.657 & 0.795 \\
\hline thb & 0.814 & 52.02 & 34.17 & 19.39 & 7.16 & 3.45 & 0.498 & 0.476 & 0.696 \\
\hline thrb & 0.848 & 40.42 & 28.04 & 19.34 & 7.45 & 3.73 & 0.530 & 0.478 & 0.674 \\
\hline try1 & 0.734 & 37.86 & 21.02 & 12.39 & 4.86 & 2.82 & 0.352 & 0.353 & 0.611 \\
\hline tryb1 & 0.646 & 16.19 & 8.77 & 7.08 & 3.46 & 2.06 & 0.229 & 0.191 & 0.342 \\
\hline tysy & 0.697 & 14.76 & 14.76 & 10.99 & 4.70 & 2.57 & 0.305 & 0.237 & 0.245 \\
\hline urok & 0.840 & 51.87 & 36.43 & 24.08 & 7.10 & 3.61 & 0.559 & 0.571 & 0.794 \\
\hline vgfr2 & 0.737 & 23.41 & 15.42 & 9.65 & 4.43 & 2.78 & 0.293 & 0.250 & 0.403 \\
\hline wee1 & 0.838 & 61.27 & 44.74 & 25.98 & 8.33 & 3.58 & 0.635 & 0.666 & 0.900 \\
\hline xiap & 0.942 & 50.42 & 51.43 & 44.95 & 11.36 & 4.55 & 0.921 & 0.946 & 0.978 \\
\hline
\end{tabular}




\section{Potential source of bias in DUD-E benchmark dataset composition due to the higher number of forms for actives than decoys}

In the DUD-E dataset, ${ }^{8}$ prepared and provided on the website as an sdf, ${ }^{9}$ actives are represented in multiple forms (tautomers, protomers, stereoisomers), whereas most decoys are present in a single form. If, as done here, the score of a molecule is taken as the score of its top-scoring form, actives have more "opportunities" to score highly than decoys. This bias should increase the expected value of the AUC above 0.5 for random selection. As the number of active and decoy forms change from target to target, the strength of this bias, and the expected AUC corresponding to random selection should also vary.

In order to understand the effect of this bias, we simulated random behavior by generating random numbers drawn from the same distribution as activities for all forms of the actives and decoys and then evaluated performance using standard performance measures. As shown for each target in Table S8, the baseline for the calculated virtual screening properties will show a bias that depends on the number of active and decoy forms. The results indicate that AUC is strongly affected, the value corresponding to random behavior is significantly above 0.5 . In contrast, the changes in BEDROC and EF metrics are negligible compared to their observed values in virtual screens (cf. Table S7). We note that, in practice, good virtual screening methods are likely to score the form (i.e., protomer, tautomer, or stereoisomer) of the active molecule with the highest activity considerably higher than other forms, making the impact of the bias on their performance as judged by AUC likely somewhat weaker than suggested by the shift in the random baseline.

In future uses of the DUD-E set, it may be worth considering approaches that substantially reduce or remove this bias. The same ligand preparation could be used for actives and decoys, 
for example. Since the average number of forms will not be exactly the same for the two classes, this will likely greatly diminish the bias but may not entirely remove it.

It is worth noting that the above bias introduces some uncertainty when comparing with the literature, as the existence and strength of the bias depend on details that are often unreported, notably how the inactive ligands were prepared and how a single score for a molecule is obtained from the scores of its various forms (taking a mean score over the various forms would be unbiased, for example). Although such information is often not explicitly provided in publications describing virtual screening methods, it seems likely that the choices we have made here are common: We believe that most publications using DUD-E used the already prepared ligand set from the DUD-E website (indeed, all the papers from which we took data for comparison that specified how ligands were prepared used this prepared set), and chose the maximum score from virtual screening as the single score for the molecule (since this is by far the most natural choice). 
Table S8. Upper bounds for random performance for different metrics on the DUD-E set. The values for each target were obtained by generating random numbers for each form of the actives and the decoys and then taking the score of the best scoring form for the evaluation of performance. The values in the table represent the mean of 100 repeats. The data marked as unbiased was obtained using the same process but only using a single form for each molecule.

\begin{tabular}{|c|c|c|c|c|c|c|c|c|}
\hline Complex & AUC & $\begin{array}{c}\mathrm{EF} \\
0.5 \%\end{array}$ & $\begin{array}{l}\text { EF } \\
1 \% \\
\end{array}$ & $\begin{array}{l}\text { EF } \\
2 \%\end{array}$ & $\begin{array}{l}\mathrm{EF} \\
8 \%\end{array}$ & $\begin{array}{c}\text { BEDROC } \\
\alpha=20\end{array}$ & $\begin{array}{c}\text { BEDROC } \\
\alpha=80.5\end{array}$ & $\begin{array}{c}\text { BEDROC } \\
\alpha=321.0\end{array}$ \\
\hline aa2ar & 0.586 & 1.69 & 1.64 & 1.67 & 1.61 & 0.092 & 0.036 & 0.026 \\
\hline abl1 & 0.588 & 1.56 & 1.63 & 1.61 & 1.51 & 0.088 & 0.036 & 0.026 \\
\hline ace & 0.646 & 2.59 & 2.51 & 2.55 & 2.30 & 0.132 & 0.057 & 0.044 \\
\hline aces & 0.562 & 1.36 & 1.44 & 1.44 & 1.41 & 0.082 & 0.032 & 0.023 \\
\hline ada & 0.620 & 2.86 & 2.60 & 2.59 & 2.19 & 0.127 & 0.057 & 0.047 \\
\hline ada17 & 0.600 & 1.62 & 1.67 & 1.75 & 1.67 & 0.095 & 0.036 & 0.024 \\
\hline adrb1 & 0.606 & 1.60 & 1.72 & 1.65 & 1.68 & 0.096 & 0.036 & 0.025 \\
\hline adrb2 & 0.612 & 1.78 & 1.73 & 1.75 & 1.77 & 0.099 & 0.037 & 0.026 \\
\hline akt1 & 0.565 & 1.50 & 1.49 & 1.44 & 1.36 & 0.081 & 0.033 & 0.026 \\
\hline akt2 & 0.576 & 1.73 & 1.53 & 1.50 & 1.49 & 0.087 & 0.036 & 0.029 \\
\hline aldr & 0.545 & 1.39 & 1.50 & 1.48 & 1.34 & 0.079 & 0.033 & 0.024 \\
\hline ampc & 0.542 & 0.99 & 1.36 & 1.13 & 1.19 & 0.071 & 0.027 & 0.020 \\
\hline andr & 0.582 & 1.84 & 1.68 & 1.68 & 1.65 & 0.096 & 0.040 & 0.033 \\
\hline aofb & 0.549 & 1.37 & 1.37 & 1.17 & 1.27 & 0.074 & 0.029 & 0.023 \\
\hline bace1 & 0.586 & 1.61 & 1.71 & 1.61 & 1.57 & 0.090 & 0.036 & 0.026 \\
\hline braf & 0.592 & 1.49 & 1.49 & 1.58 & 1.59 & 0.090 & 0.033 & 0.023 \\
\hline cah2 & 0.576 & 1.60 & 1.59 & 1.56 & 1.52 & 0.088 & 0.034 & 0.025 \\
\hline casp3 & 0.591 & 1.61 & 1.61 & 1.59 & 1.62 & 0.094 & 0.038 & 0.028 \\
\hline $\mathrm{cdk} 2$ & 0.592 & 1.66 & 1.71 & 1.64 & 1.55 & 0.092 & 0.037 & 0.028 \\
\hline comt & 0.657 & 1.63 & 2.21 & 2.21 & 1.97 & 0.109 & 0.038 & 0.019 \\
\hline cp2c9 & 0.561 & 1.31 & 1.40 & 1.40 & 1.43 & 0.081 & 0.030 & 0.020 \\
\hline cp3a4 & 0.611 & 1.97 & 1.90 & 1.88 & 1.88 & 0.105 & 0.040 & 0.028 \\
\hline csflr & 0.605 & 1.69 & 1.71 & 1.56 & 1.59 & 0.089 & 0.033 & 0.023 \\
\hline cxcr4 & 0.672 & 3.26 & 3.07 & 2.75 & 2.52 & 0.138 & 0.054 & 0.039 \\
\hline def & 0.580 & 1.47 & 1.57 & 1.55 & 1.52 & 0.088 & 0.036 & 0.027 \\
\hline dhil & 0.566 & 1.52 & 1.49 & 1.47 & 1.44 & 0.084 & 0.033 & 0.026 \\
\hline dpp4 & 0.623 & 1.97 & 1.96 & 1.95 & 1.81 & 0.102 & 0.038 & 0.025 \\
\hline $\mathrm{drd} 3$ & 0.611 & 1.64 & 1.71 & 1.74 & 1.68 & 0.095 & 0.036 & 0.023 \\
\hline dyr & 0.639 & 2.26 & 2.25 & 2.21 & 2.02 & 0.114 & 0.044 & 0.030 \\
\hline egfr & 0.577 & 1.54 & 1.51 & 1.55 & 1.46 & 0.084 & 0.033 & 0.023 \\
\hline
\end{tabular}




\begin{tabular}{|c|c|c|c|c|c|c|c|c|}
\hline esr 1 & 0.568 & 1.54 & 1.46 & 1.55 & 1.51 & 0.088 & 0.036 & 0.028 \\
\hline esr2 & 0.570 & 1.59 & 1.62 & 1.64 & 1.50 & 0.089 & 0.038 & 0.029 \\
\hline fa10 & 0.544 & 1.34 & 1.33 & 1.31 & 1.29 & 0.077 & 0.032 & 0.025 \\
\hline fa7 & 0.589 & 1.56 & 1.66 & 1.62 & 1.54 & 0.091 & 0.038 & 0.032 \\
\hline fabp4 & 0.535 & 1.15 & 1.38 & 1.37 & 1.22 & 0.072 & 0.029 & 0.020 \\
\hline fak1 & 0.516 & 1.34 & 1.27 & 1.16 & 1.08 & 0.065 & 0.028 & 0.022 \\
\hline fgfr1 & 0.588 & 1.56 & 1.60 & 1.68 & 1.53 & 0.089 & 0.036 & 0.025 \\
\hline fkbla & 0.659 & 2.01 & 2.02 & 2.22 & 2.17 & 0.126 & 0.052 & 0.036 \\
\hline fnta & 0.685 & 2.63 & 2.68 & 2.67 & 2.46 & 0.135 & 0.050 & 0.031 \\
\hline fpps & 0.691 & 2.22 & 2.12 & 2.10 & 2.13 & 0.115 & 0.038 & 0.020 \\
\hline gcr & 0.623 & 2.23 & 2.11 & 2.06 & 1.93 & 0.112 & 0.047 & 0.037 \\
\hline $\mathrm{glcm}$ & 0.672 & 4.83 & 5.08 & 4.68 & 3.23 & 0.188 & 0.095 & 0.074 \\
\hline gria2 & 0.620 & 1.69 & 1.75 & 1.76 & 1.75 & 0.098 & 0.035 & 0.022 \\
\hline grik1 & 0.571 & 1.51 & 1.58 & 1.59 & 1.46 & 0.085 & 0.034 & 0.024 \\
\hline hdac2 & 0.545 & 1.43 & 1.44 & 1.40 & 1.28 & 0.075 & 0.032 & 0.025 \\
\hline hdac8 & 0.557 & 1.25 & 1.31 & 1.38 & 1.30 & 0.076 & 0.029 & 0.019 \\
\hline hivint & 0.606 & 1.63 & 1.84 & 2.00 & 1.83 & 0.103 & 0.040 & 0.024 \\
\hline hivpr & 0.633 & 2.53 & 2.54 & 2.49 & 2.20 & 0.125 & 0.052 & 0.038 \\
\hline hivrt & 0.562 & 1.50 & 1.53 & 1.48 & 1.46 & 0.086 & 0.035 & 0.027 \\
\hline hmdh & 0.595 & 1.64 & 1.47 & 1.57 & 1.61 & 0.095 & 0.038 & 0.030 \\
\hline hs90a & 0.556 & 1.49 & 1.44 & 1.54 & 1.41 & 0.083 & 0.035 & 0.027 \\
\hline hxk4 & 0.560 & 1.72 & 1.53 & 1.44 & 1.41 & 0.084 & 0.037 & 0.033 \\
\hline igflr & 0.574 & 1.54 & 1.49 & 1.48 & 1.42 & 0.083 & 0.033 & 0.025 \\
\hline inha & 0.570 & 1.53 & 1.48 & 1.68 & 1.51 & 0.091 & 0.038 & 0.030 \\
\hline ital & 0.571 & 1.74 & 1.66 & 1.58 & 1.54 & 0.089 & 0.036 & 0.026 \\
\hline jak2 & 0.567 & 1.46 & 1.44 & 1.37 & 1.36 & 0.079 & 0.031 & 0.023 \\
\hline kif11 & 0.592 & 1.68 & 1.74 & 1.65 & 1.55 & 0.091 & 0.037 & 0.028 \\
\hline kit & 0.573 & 1.47 & 1.55 & 1.43 & 1.43 & 0.082 & 0.032 & 0.023 \\
\hline kith & 0.597 & 2.62 & 2.53 & 2.20 & 2.00 & 0.120 & 0.057 & 0.051 \\
\hline $\mathrm{kpcb}$ & 0.578 & 1.78 & 1.82 & 1.72 & 1.63 & 0.092 & 0.037 & 0.026 \\
\hline lck & 0.580 & 1.69 & 1.65 & 1.58 & 1.54 & 0.087 & 0.034 & 0.026 \\
\hline lkha4 & 0.556 & 1.44 & 1.41 & 1.42 & 1.36 & 0.080 & 0.033 & 0.026 \\
\hline mapk2 & 0.577 & 1.76 & 1.65 & 1.63 & 1.62 & 0.092 & 0.036 & 0.027 \\
\hline $\mathrm{mcr}$ & 0.627 & 1.71 & 1.88 & 1.99 & 1.91 & 0.111 & 0.046 & 0.032 \\
\hline met & 0.563 & 1.37 & 1.22 & 1.33 & 1.36 & 0.078 & 0.028 & 0.021 \\
\hline $\mathrm{mk} 01$ & 0.594 & 1.76 & 1.79 & 1.79 & 1.61 & 0.095 & 0.040 & 0.030 \\
\hline $\mathrm{mk} 10$ & 0.607 & 1.89 & 1.79 & 1.74 & 1.67 & 0.096 & 0.038 & 0.028 \\
\hline $\mathrm{mk} 14$ & 0.572 & 1.61 & 1.56 & 1.50 & 1.45 & 0.084 & 0.033 & 0.026 \\
\hline mmp13 & 0.601 & 1.69 & 1.69 & 1.71 & 1.67 & 0.095 & 0.037 & 0.026 \\
\hline $\mathrm{mp} 2 \mathrm{k} 1$ & 0.621 & 1.82 & 1.60 & 1.73 & 1.75 & 0.099 & 0.037 & 0.027 \\
\hline nos1 & 0.660 & 2.12 & 2.09 & 2.20 & 2.15 & 0.117 & 0.042 & 0.027 \\
\hline nram & 0.638 & 2.11 & 2.18 & 2.14 & 2.04 & 0.117 & 0.047 & 0.034 \\
\hline
\end{tabular}




\begin{tabular}{|c|c|c|c|c|c|c|c|c|}
\hline pa2ga & 0.531 & 1.06 & 1.17 & 1.20 & 1.19 & 0.072 & 0.029 & 0.021 \\
\hline parp1 & 0.563 & 1.43 & 1.41 & 1.37 & 1.34 & 0.079 & 0.031 & 0.023 \\
\hline pde5a & 0.600 & 1.78 & 1.76 & 1.70 & 1.64 & 0.094 & 0.036 & 0.025 \\
\hline pgh1 & 0.538 & 1.26 & 1.25 & 1.23 & 1.21 & 0.072 & 0.029 & 0.021 \\
\hline pgh2 & 0.528 & 1.18 & 1.15 & 1.15 & 1.20 & 0.071 & 0.028 & 0.021 \\
\hline plk1 & 0.563 & 1.40 & 1.37 & 1.43 & 1.37 & 0.079 & 0.031 & 0.022 \\
\hline pnph & 0.629 & 2.19 & 2.08 & 2.02 & 1.90 & 0.109 & 0.043 & 0.034 \\
\hline ppara & 0.561 & 1.53 & 1.51 & 1.47 & 1.39 & 0.083 & 0.036 & 0.030 \\
\hline ppard & 0.517 & 1.06 & 1.02 & 1.07 & 1.06 & 0.064 & 0.026 & 0.020 \\
\hline pparg & 0.564 & 1.39 & 1.35 & 1.39 & 1.39 & 0.082 & 0.033 & 0.025 \\
\hline prgr & 0.575 & 1.54 & 1.47 & 1.50 & 1.46 & 0.086 & 0.035 & 0.028 \\
\hline ptn1 & 0.580 & 1.75 & 1.57 & 1.64 & 1.56 & 0.091 & 0.038 & 0.031 \\
\hline pur2 & 0.682 & 4.06 & 3.79 & 3.72 & 2.95 & 0.175 & 0.087 & 0.077 \\
\hline pygm & 0.572 & 0.84 & 0.91 & 1.25 & 1.35 & 0.079 & 0.029 & 0.017 \\
\hline pyrd & 0.528 & 1.24 & 1.14 & 1.12 & 1.12 & 0.066 & 0.026 & 0.020 \\
\hline reni & 0.636 & 3.80 & 3.64 & 3.28 & 2.46 & 0.143 & 0.069 & 0.054 \\
\hline rock1 & 0.616 & 2.12 & 2.13 & 2.12 & 1.88 & 0.109 & 0.045 & 0.032 \\
\hline rxra & 0.514 & 0.87 & 1.01 & 1.12 & 1.10 & 0.065 & 0.025 & 0.017 \\
\hline sahh & 0.621 & 3.03 & 3.12 & 2.87 & 2.27 & 0.133 & 0.066 & 0.057 \\
\hline src & 0.584 & 1.55 & 1.53 & 1.53 & 1.48 & 0.085 & 0.032 & 0.024 \\
\hline $\operatorname{tgfr} 1$ & 0.625 & 2.14 & 2.07 & 2.02 & 1.86 & 0.107 & 0.043 & 0.034 \\
\hline thb & 0.586 & 1.81 & 1.73 & 1.67 & 1.56 & 0.088 & 0.034 & 0.025 \\
\hline thrb & 0.605 & 1.76 & 1.75 & 1.77 & 1.70 & 0.098 & 0.040 & 0.029 \\
\hline try1 & 0.588 & 1.75 & 1.70 & 1.67 & 1.59 & 0.093 & 0.038 & 0.029 \\
\hline tryb1 & 0.527 & 1.24 & 1.20 & 1.21 & 1.16 & 0.069 & 0.029 & 0.023 \\
\hline tysy & 0.608 & 2.46 & 2.52 & 2.57 & 2.08 & 0.121 & 0.054 & 0.040 \\
\hline urok & 0.604 & 1.87 & 1.88 & 1.77 & 1.72 & 0.099 & 0.039 & 0.029 \\
\hline vgfr2 & 0.573 & 1.52 & 1.44 & 1.42 & 1.42 & 0.082 & 0.032 & 0.023 \\
\hline wee1 & 0.548 & 1.33 & 1.37 & 1.35 & 1.27 & 0.075 & 0.030 & 0.022 \\
\hline xiap & 0.539 & 1.26 & 1.28 & 1.34 & 1.33 & 0.079 & 0.032 & 0.023 \\
\hline Mean & 0.588 & 1.76 & 1.76 & 1.74 & 1.63 & 0.094 & 0.038 & 0.028 \\
\hline Median & 0.583 & 1.62 & 1.61 & 1.61 & 1.55 & 0.089 & 0.036 & 0.026 \\
\hline Unbiased & 0.500 & 1.00 & 1.00 & 1.00 & 1.00 & 0.059 & 0.022 & 0.016 \\
\hline
\end{tabular}




\section{Supporting References}

1. Molecular Operating Environment (MOE), version 2015.10. Chemical Computing Group, Montreal, Canada, 2015.

2. Wieder, M.; Garon, A.; Perricone, U.; Boresch, S.; Seidel, T.; Almerico, A. M.; Langer, T. Common hits approach: combining pharmacophore modeling and molecular dynamics simulations. J. Chem. Inf. Model. 2017, 57(2), 365-385.

3. Chaput, L.; Martinez-Sanz, J.; Saettel, N.; Mouawad, L. Benchmark of four popular virtual screening programs: construction of the active/decoy dataset remains a major determinant of measured performance. J. Cheminform. 2016, 8(1), 56-72.

4. Ruiz-Carmona, S.; Alvarez-Garcia, D.; Foloppe, N.; Garmendia-Doval, A. B.; Juhos, S.; Schmidtke, P.; Barril, X.; Hubbard, R. E.; Morley, S. D. rDock: a fast, versatile, and open source program for docking ligands to proteins and nucleic acids. PLoS Comput. Biol. 2014, 10(4), e1003571.

5. Zhang, X.; Wong, S. E.; Lightstone, F. C. Toward fully automated high performance computing drug discovery: a massively parallel virtual screening pipeline for docking and molecular mechanics/generalized Born surface area rescoring to improve enrichment. $J$. Chem. Inf. Model. 2014, 54(1), 324-337.

6. Coleman, R. G.; Sterling, T.; Weiss, D. R. SAMPL4 \& DOCK3.7: lessons for automated docking procedures. J. Comput. Aided Mol. Des. 2014, 28(3), 201-209.

7. Tran-Nguyen, V.K.; Da Silva, F.; Bret, G.; Rognan, D. All in one: cavity detection, druggability estimate, cavity-based pharmacophore perception, and virtual screening. $J$. Chem. Inf. Model. 2019, 59, 573-585.

8. Mysinger, M.M.; Carchia, M.; Irwin, J.J.; Shoichet, B.K. Directory of Useful Decoys, Enhanced (DUD-E): better ligands and decoys for better benchmarking. J. Med. Chem. 2012, 55(14), 6582-6594. 
9. DUD-E, a database of useful decoys. http://dude.docking.org/ (accessed February 23, 2017).

10. Coleman, R.G.; Sterling, T.; Weiss, D.R. SAMPL4 \& DOCK3.7: lessons for automated docking procedures. J. Comput. Aided Mol. Des. 2014, 28(3), 201-209. 